\title{
The Influence of Contrast Adaptation on Color
}

\author{
Appearance \\ MICHAEL A. WEBSTER, ${ }^{*}$ J. D. MOLLON* \\ Received 23 February 1993; in revised form 14 September 1993
}

\begin{abstract}
Most models of color vision assume that signals from the three classes of cone receptor are recoded into only three independent post-receptoral channels: one that encodes luminance and two that encode color. Stimuli that are equated for their effects on two of the channels should be discriminable only to the remaining channel, and are thus assumed to isolate the responses of single channels. We used an asymmetric matching task to examine whether such models can account for changes in color appearance following adaptation to contrast- to temporal variations in luminance and chromaticity around a fixed mean luminance and chromaticity. The experiments extend to suprathreshold color appearance the threshold adaptation paradigm of Krauskopf, Williams and Heeley I(1982) Vision Research, 32, 1123-1131]. Adaptation changes the perceived color of chromatic test stimuli both by reducing their saturation (contrast) and by changing their hue (direction within the equiluminant plane). The saturation losses are largest for test stimuli that lie along the chromatic axis defining the adapting modulation, while the hue changes are rotations away from the adapting direction and toward an orthogonal direction within the $S$ and $L-M$ plane. Similar selective changes in both perceived color and perceived lightness occur following adaptation to stimuli that covary in luminance and chromaticity. The selectivity of the aftereffects for multiple directions within color-luminance space is inconsistent with sensitivity changes in only three independent channels. These aftereffects suggest instead that color appearance depends on channels that can be selectively tuned to any color-luminance direction, and that there are no directions that invariably isolate responses in only a single channel. We use the perceived color changes to examine the spectral sensitivities of the chromatic channels and to estimate the distribution of channels. We also examine how adaptation alters the contrast-response function, how it affects reaction times for luminance and chromatic contrast, the extent to which the aftereffects exhibit interocular transfer, and the way in which the perceived color changes differ from those induced by conventional light adaptation.
\end{abstract}

Color appearance Luminance and chromatic channels Contrast adaptation Cardinal color directions Color-luminance interactions

\section{INTRODUCTION}

Most models of color vision assume only three independent post-receptoral channels one that encodes luminance and two that encode color (Lennie \& D'Zmura, 1988). The luminance channel depends on inputs of the same sign from different cone classes, while the chromatic channels draw inputs of opposite sign. However, the number and nature of the post-receptoral transformations of the cone signals remain ill established. The phenomenal appearance of chromatic stimuli suggested two "second-stage" chromatic channels whose responses correspond to sensations of red-green or blue-yellow (Hurvich \& Jameson, 1957). However, measures of threshold sensitivity for chromatic stimuli reveal postreceptoral signals that are instead organized along two chromatic dimensions that correspond to (a) opposing

*Department of Experimental Psychology, University of Cambridge, Downing Street. Cambridge CB2 3EB, England. signals from the long- and medium-wavelength cones $(\mathrm{L}-\mathrm{M})$, or (b) signals from short-wavelength cones opposed by a combination of signals from the $L$ and $M$ cones [S - (L + M), or S] (Le Grand, 1949; Krauskopf, Williams \& Heeley, 1982). Stimuli that isolate the latter dimension differ markedly from a pure blue-yellow variation. The $\mathrm{S}$ dimension may represent an ancient dichromatic color system that compared signals in the $S$ cones with a single, ancestral medium- or longwavelength cone type, while the $\mathrm{L}-\mathrm{M}$ dimension may represent a modern color subsystem that arose after differentiation of the genes encoding the $\mathrm{L}$ and $\mathrm{M}$ photopigments (Mollon, 1989). The chromatic selectivities of cells in the lateral geniculate are clustered along these two dimensions (Derrington, Krauskopf \& Lennie, 1984). Psychophysical studies imply additionally a third, luminance dimension, which depends primarily on the sum of the signals in the $L$ and $M$ cones, with little contribution from the $\mathrm{S}$ cones (Lennie, Pokorny \& Smith, 1993). However, cells in the parvocellular 
pathway (which is the likely substrate for color appearance) do not show a clear separation between luminance and chromatic sensitivity (Wiesel \& Hubel, 1966; De Valois, Snodderly, Yund \& Hepler, 1977; Derrington et al., 1984).

In order to explore how the triplet of cone signals are organized within the post-receptoral mechanisms that contribute to color appearance, we have examined how color appearance is altered after adaptation to contrast, i.e. to variations in luminance and chromaticity around a fixed average luminance and chromaticity. Prior adaptation to contrast elevates thresholds for detecting luminance or chromatic contrast (Blakemore \& Campbell, 1969; Benzschawel \& Guth, 1982; Krauskopf et al., 1982; Bradley, Switkes \& De Valois, 1988), reduces the apparent contrast of suprathreshold stimuli (Blakemore, Muncey \& Ridley, 1971; Pantle, 1972; Georgeson, 1985), and can induce qualitative changes in the appearance of patterns [as in the aftereffects of motion (Wohlgemuth, 1911), tilt (Gibson \& Radner, 1937), and spatial frequency (Blakemore \& Sutton, 1969)]. These aftereffects are thought to reflect sensitivity changes at a cortical locus, and are distinct from the light adaptation effects that have conventionally been studied in color vision (Jameson \& Hurvich, 1972).

Contrast adaptation has been widely used as a tool for probing the number and selectivity of the mechanisms involved in form or motion perception, and was first used to examine the spectral sensitivities of the channels mediating color vision by Krauskopf and colleagues (Krauskopf et al., 1982; Krauskopf, Williams, Mandler
\& Brown, 1986b) and by Guth and colleagues (Benzschawel \& Guth, 1982; Guth, 1982; Moxley, Guth \& Geisler, 1979; Moxley \& Guth, 1981; Guth \& Moxley. 1982). Krauskopf et al. examined how adaptation to temporal modulations along different directions in colorluminance space altered thresholds for detecting brief changes in a field's luminance and chromaticity. They found that changes in sensitivity were primarily selective for three "cardinal" directions corresponding to an achromatic axis (along which only luminance varies) and to the $\mathrm{L}-\mathrm{M}$ and $\mathrm{S}$ chromatic axes [Fig. I(A)]. For example, adaptation to modulations along the $L-M$ axis greatly elevated thresholds for detecting a color change along the $\mathrm{L}-\mathrm{M}$ axis, but had little effect on the thresholds for detecting changes along the $\mathrm{S}$ or luminance axes. Their results therefore suggested that threshold sensitivity depends on three primary types of adaptable channel that are isolated by these three types of stimulus variation. $\Lambda$ daptation to directions that were intermediate to the three cardinal axes produced much more general losses in sensitivity. However, analyses of the threshold changes within the equiluminant plane demonstrated that the sensitivity losses always showed some selectivity for the chromatic adapting direction, suggesting that there were also additional chromatic channels tuned to intermediate chromatic directions (Krauskopf et al., 1986b). Multiple chromatic mechanisms have also been invoked to account for results in a variety of other psychophysical tasks (D'Zmura, 1991; Flanagan, Cavanagh \& Favreau, 1990; Guth, 1982; Krauskopf et al., 1986b; Krauskopf \& Gegenfurtner,
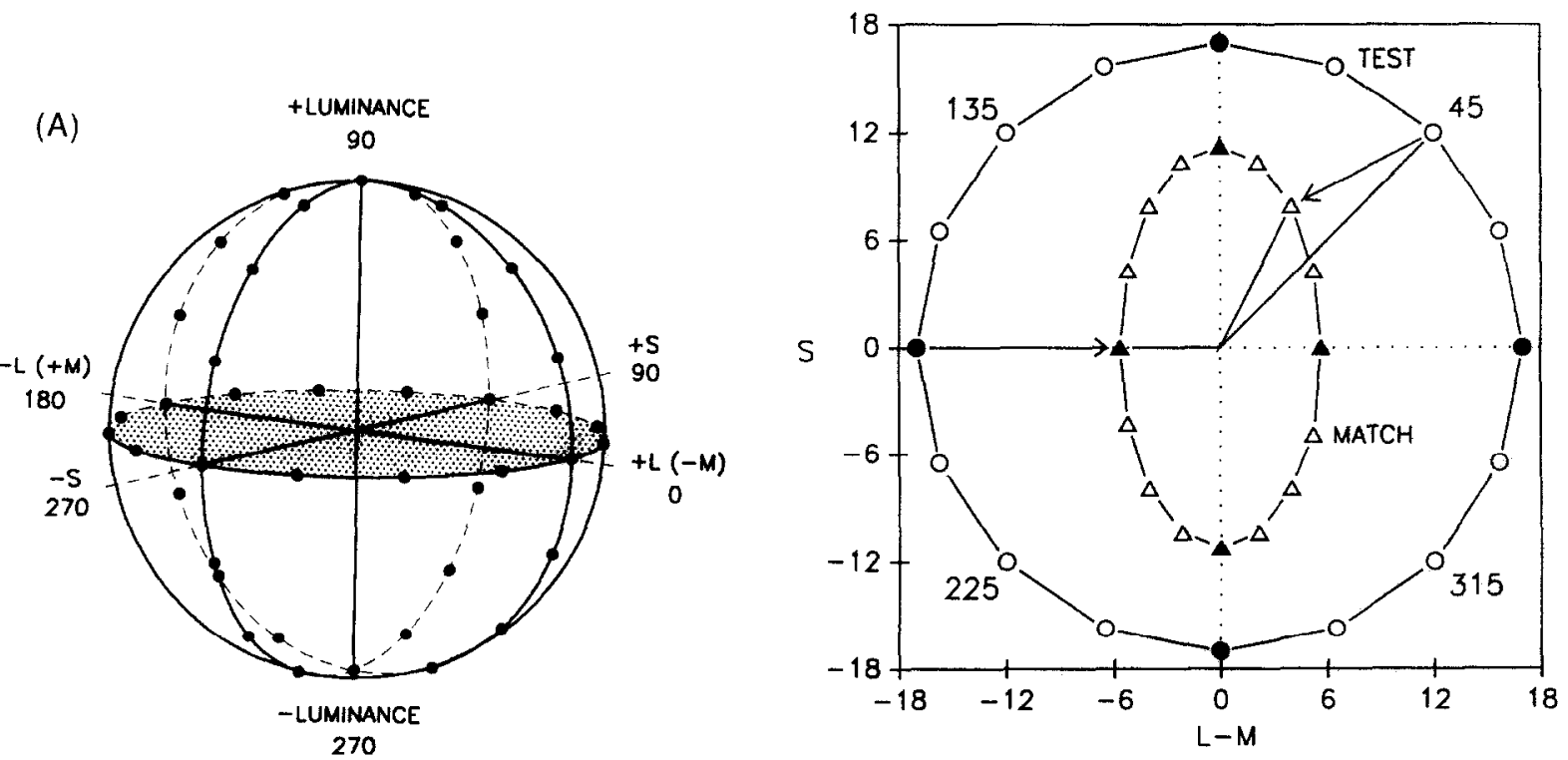

FIGURE 1. (A) A color space defined by the luminance axis and the $\mathrm{S}$ and $\mathrm{L}-\mathrm{M}$ chromatic axes (following MacLeod \& Boynton, 1979; Derrington et al., 1984). Symbols plot the set of test stimuli examined. (B) Color changes within the equiluminant plane predicted from independent adaptation in two channels sensitive to $S$ or $L-M$ stimulus variations. The outer circles plot the chromaticities of 16 test stimuli that have the same contrast, but lie along different directions from the achromatic origin. The inner triangles plot the perceived chromaticity of the test stimuli following adaptation to a stimulus that reduces sensitivity in the $L-M$ channel more than the $S$ channel. This produces the largest contrast loss in stimuli along the $L-M$ axis, and biases the perceived direction of stimuli away from the $L-M$ axis and toward the $S$ axis. Stimuli along the $\mathrm{L}-\mathrm{M}$ or $\mathrm{S}$ axes may show a change in perceived contrast, but should not show a change in perceived direction (solid triangles). 
1992; Krauskopf, Zaidi \& Mandler, 1986a; Zaidi \& Halevy, 1993). Yet under other conditions performance can be well described by the independent signals along the $S$ and $L-M$ axes (Boynton \& Kambe, 1980; Cole, Hine \& Mcllhagga, 1993; Krauskopf \& Farell, 1990; Krauskopf \& Gegenfurtner, 1992; Le Grand, 1949; Nagy \& Sanchez, 1990; Stromeyer \& Lee, 1988).

We have extended the adaptation paradigm and logic of the Krauskopf et al. $(1982,1986 \mathrm{~b})$ studies to examine how contrast adaptation influences the perception of suprathreshold colors. Our study is similar in rationale to experiments on adaptation-induced changes in perceived hue reported by Guth and his colleagues (Moxley et al., 1979; Moxley \& Guth, 1981; Guth, 1982; Guth \& Moxley, 1982) and Flanagan, Cavanagh and Crasini (1989). Models based on three independent post-receptoral channels make testable predictions about how adaptation should alter color appearance. Figure 1(B) illustrates these predictions for a set of equiluminant test stimuli encoded by the responses in two chromatic channels selectively sensitive to the $\mathrm{L}-\mathrm{M}$ or $\mathrm{S}$ chromatic axes. In this plane the contrast of a stimulus depends on how far it is from the achromatic origin and corresponds roughly to perceived saturation, while the perceived hue of the stimulus corresponds roughly to its direction from the origin. Adaptation could change the perceived contrast and direction of test stimuli by changing sensitivity in one or both of the channels. (We assume that contrast adaptation alters only the responsiveness, or contrast gain, of the channels, without affecting their mean response, as we verify below.) If these sensitivity changes are independent, then the least or greatest change in perceived contrast should always occur along only two directions (corresponding to the two directions that isolate the two channels: the $\mathrm{L}-\mathrm{M}$ and $S$ axes in this example). Moreover, equivalent changes in color appearance should follow adaptation to any stimuli that have the same component contrast within each channel. In this example such stimuli correspond to any pair of adapting axes that are symmetrical about the $L-M$ and $S$ axes (e.g. axes of 45-225 and $135-315 \mathrm{deg}$ ). These stimulus pairs differ only in how the $\mathrm{L}-\mathrm{M}$ and $\mathrm{S}$ component contrasts are combined. Most stimuli will produce responses in both channels. For such stimuli a change in their perceived direction should arise whenever adaptation alters sensitivity in one of the channels more than the other. For example, if the $\mathrm{L}-\mathrm{M}$ channel is adapted more than the S channel, then this should bias the perceived hue of test stimuli toward the $\mathrm{S}$ axis. These hue changes are analogous to spatial adaptation phenomena such as the tilt aftereffect, for they represent "tilts" in the perceived orientation of axes within color space. However, since some stimulus axes isolate the response of only a single chromatic channel, and should continue to isolate the same channel after adaptation, then the perceived direction of these axes should remain invariant. The changes in the perceived hue of all other test axes should always be rotations toward one of these invariant axes and away from the other (depending on which of the two channels is adapted more).

Specifically how adaptation alters color appearance will depend on such factors as the spectral sensitivities of the channels, how contrast adaptation alters the channel responses, and how these responses are pooled. However, the above predictions are a general property of models in which changes in contrast gain occur independently in three post-receptoral channels that linearly combine the cone signals. We show that these predictions fail to describe the changes in color appearance following adaptation. Adaptation to any direction within color-luminance space produces suprathreshold color changes that are selective for the adapting direction. Thus color appearance depends either on many more than three post-receptoral channels with different tuning functions, or on channels that can change their tuning functions through adaptation. A preliminary account of this work was given in Webster and Mollon (1991).

\section{METHOD}

Stimuli were presented on a $60 \mathrm{~Hz}$ interlaced Barco CD 351 color monitor, controlled by a Sigma Electronic Systems 5688 graphics system. Luminances of the monitor guns were calibrated with a Minolta Chromameter, and linearized through look-up tables. Chromaticities (CIE, 1931) of the three guns were initially estimated with a Lovibond Tintometer to be $(x=0.617, y=0.353)$ for the red gun, $(x=0.290, y=0.599)$ for the green, and $(x=0.152, y=0.063)$ for the blue. Subsequent cstimates with the chromameter were very similar. The graphics system allowed intensities of the red, green, and blue guns to be specified to an accuracy of 8 bits/gun. For stimuli of low contrast, the color resolution of the display was increased by presenting the stimuli on only a proportion of the scan lines, with the remaining lines maintained at zero-contrast (equal to the luminance and chromaticity of the background). For example, test and matching stimuli were typically presented only on alternate scan lines, increasing the effective resolution to 9 bits/gun. This "halftoning" was not visible to the observer at the viewing distances and low contrasts at which it was introduced. The actual chromaticities due to quantization of the gun luminances differed only slightly from the nominal chromaticities plotted (e.g. r.m.s. error $=0.27$ threshold units for the individual matches illustrated in Fig. 3, or 0.12 threshold units for the mean of the matches, values that are 3-8 times smaller, respectively, than the r.m.s. variability in the nominal matches).

Stimuli were defined in terms of a three-dimensional color space whose principal axes isolate variations either in luminance, in $S$ cone excitation at constant luminance, or in the ratio of $\mathrm{L}$ to $\mathrm{M}$ cone excitation at constant luminance (MacLeod \& Boynton, 1979; Derrington et al., 1984). The axis that isolates luminance variations is the achromatic axis, along which signals from all three cone types vary in constant proportion. Thus it does not 
(A)

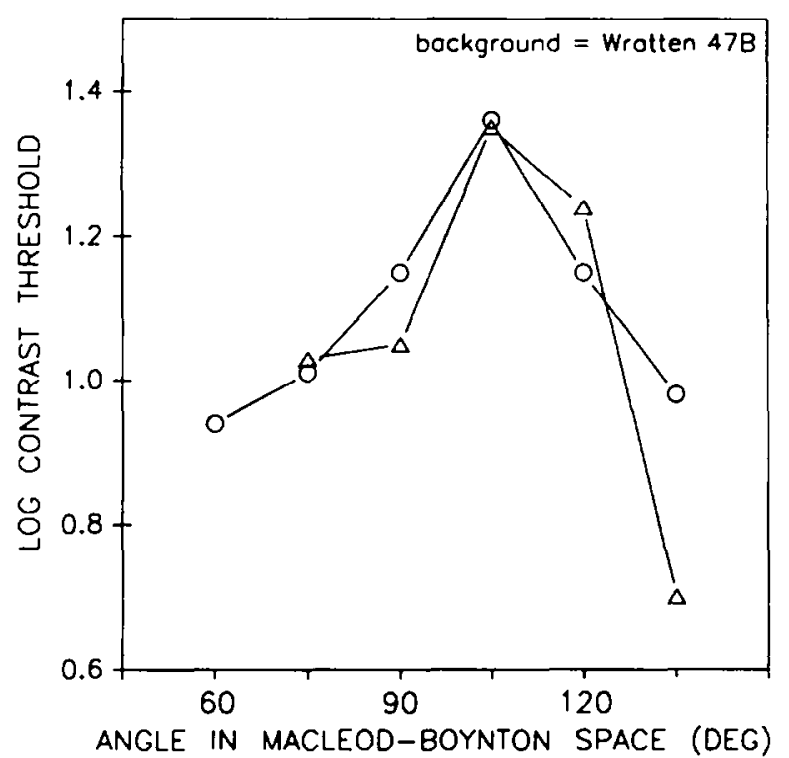

(B)

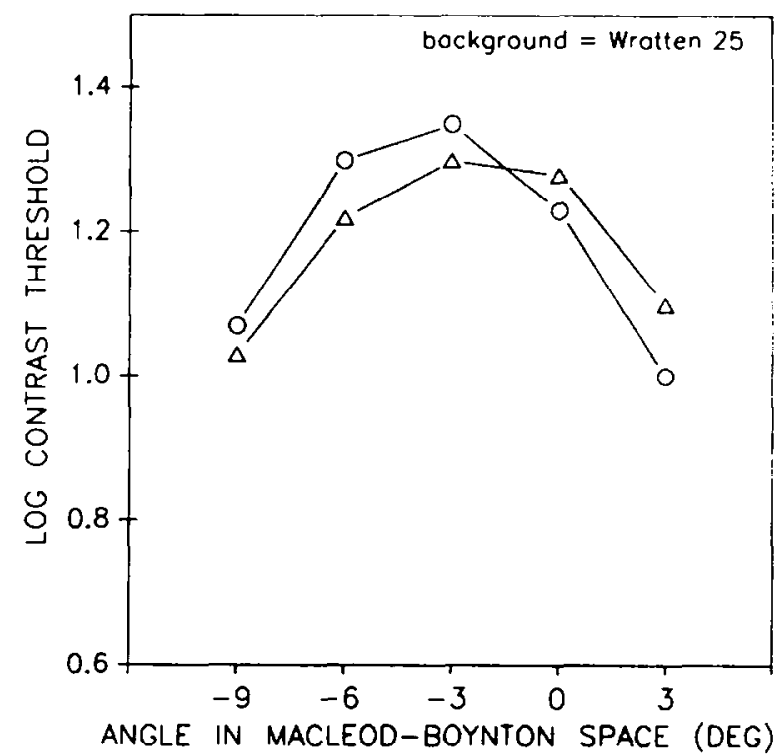

FIGURE 2. Contrast thresholds for detecting color changes along different axes of the MacLeod Boynton chromaticity diagram, in the presence of short-wavelength (A) or long-wavelength (B) adapting backgrounds. Test angles of 0 and 90 deg correspond to the $+\mathrm{L}(-\mathrm{M})$ and $+\mathrm{S}$ axes of the standard observer. In each figure the two sets of data points are for chromatic cxcursions along opposite poles of the same axis [e.g. $90 \mathrm{deg}$ (circles) and $270 \mathrm{deg}$ (triangles), or 0 deg (circles) and 180 deg (triangles)]. The relative scaling of these axes in the MacLeod . Boynton diagram requires that finer angles be sampled around the $L-M$ axis. For example. rescaling the $S$ and $L-M$ axes to equate threshold distances along the two axes [as defined by equation (1)] changes the $15 \mathrm{deg}$ intervals around the $S$ axis to roughly $7.5 \mathrm{deg}$, while changing the 3 deg intervals around the $\mathrm{L}-\mathrm{M}$ axis to roughly $6 \mathrm{deg}$ [see equation (2)].

correspond to the preferred direction of a luminance mechanism that draws inputs only from the $L$ and $M$ cones. Similarly, the preferred directions of chromatic channels will in general not lie within the equiluminant plane (e.g. see Flanagan et al., 1990; Cole et al., 1993). As we noted, these three axes correspond to the three cardinal directions identified by Krauskopf et al. (1982). The space, illustrated in Fig. 1(A), was empirically derived in three steps. First, the equiluminant plane was defined by flicker-photometrically equating the luminances of each of the monitor guns to a white reference luminance. Second, following the procedure of Webster, De Valois and Switkes (1990), we estimated the chromatic variations within this plane that changed only signals in the $\mathrm{S}$ cones or only the ratio of signals in the $\mathrm{L}$ and $\mathrm{M}$ cones. The contrast thresholds for detecting a change in color from the white background were measured along different chromatic directions chosen to span the nominal $S$ and $L-M$ axes of the MacLeod-Boynton chromaticity diagram. Thresholds were measured before or after superposing a uniform chromatic-adapting background (from a tungsten projector with gelatin filter viewed through a half-silvered mirror). Addition of a short-wavelength background (Wratten 47B) selectively reduces sensitivity in the $S$ cones and should therefore produce the largest threshold change for the chromatic direction that isolates $\mathrm{S}$ cone responses. On the other hand, a long-wavelength background (Wratten 25) selectively changes sensitivity in the $\mathrm{L}$ and $\mathrm{M}$ cones and should therefore produce the largest threshold elevation for the chromatic direction that can be detected by signals only in the $\mathrm{L}$ and $\mathrm{M}$ cones. Figure
2 shows that the $S$ and $L-M$ axes defined in this way corresponded to angles of roughly $105 \mathrm{deg}$ (S) and $-3 \operatorname{deg}(\mathrm{L}-\mathrm{M})$ within the MacLeod-Boynton space. and thus differed slightly from the 90 and 0 deg axes defining the standard observer (differences that could arise from small errors in the estimated luminances or chromaticities of the monitor phosphors). Very similar estimates were obtained for four additional subjects as part of a separate study. Finally, contrast thresholds were measured along the $S, L-M$ and achromatic axes for neutral adaptation (to the unmodulated white background). The thresholds were estimated for stimuli that had the same spatial and temporal profile as the test and matching stimuli used in the contrast adaptation measurements. These detection thresholds were used to equate the nominal contrasts along the three different axes. The resulting axes were defined to intersect at an origin corresponding to the mean color (equivalent to illuminant $\mathrm{C}$ ) and luminance $\left(27.5 \mathrm{~cd} / \mathrm{m}^{2}\right)$ of the display, and scaled contrasts along the three axes in terms of multiples of the contrast detection thresholds. These times-threshold coordinates are related to the $r, b$ coordinates in the MacLeod-Boynton chromaticity diagram and to luminance Michelson-contrast through the following equations:

$$
\begin{aligned}
r_{\mathrm{mb}}= & 0.000653(\mathrm{~L}-\mathrm{M})_{\mathrm{th}}-0.000087 \mathrm{~S}_{\mathrm{th}}+0.6568 \\
b_{\mathrm{mb}}= & -0.0000343(\mathrm{~L}-\mathrm{M})_{\mathrm{th}} \\
& +0.000323 \mathrm{~S}_{\mathrm{th}}+0.01825 \\
\mathrm{LUM}_{\mathrm{m}}= & 0.0175 \mathrm{LUM}_{\mathrm{th}}
\end{aligned}
$$


where $(L-M)_{t h}, S_{t h}$, and $L_{U M}$ are the multiples of threshold contrasts along the cardinal axes. [Note that our $\mathrm{S}$ axis is inverted relative to the $\mathrm{S}$ axis of Derrington et al. (1984), so that for a given luminance positive values of $S_{\mathrm{th}}$ correspond to greater $\mathrm{S}$ cone excitation than the background chromaticity.] Subsequent measures of the adaptation effects were consistent with our initial estimates of the equiluminant plane and cardinal axes, but suggested a revised scaling of the relative signals along the two chromatic axes (as we discuss below).

Adaptation effects were measured within the three planes that isolated different pairs of cardinal axes. Within each plane a set of either 8 or 16 test stimuli were examined. The tests had a contrast of $17 \times$ threshold and were spaced around a circle at equal intervals of 45 or $22.5 \mathrm{deg}$. The adaptation stimuli were defined by $1 \mathrm{~Hz}$ sinusoidal variations along the same eight axes (again separated by 45 or $22.5 \mathrm{deg}$ ), and varied over a range of $\pm 48 \times$ threshold. All adaptation stimuli thus had the same time-averaged luminance and chromaticity (equivalent to the background luminance and chromaticity, and to the mean of the 16 test stimulus coordinates).

Observers viewed the display monocularly from distances of 2.5 or $3.7 \mathrm{~m}$. Both the adapting and test stimuli were presented in a uniform 2 deg field, centered $1.2 \mathrm{deg}$ to one side of a central cross, which observers fixated throughout. (In some conditions the field was instead presented above or below fixation.) Narrow black borders delimited the field from a surround of the same average luminance and chromaticity. Observers initially adapted for $180 \mathrm{sec}$. 0.5 -sec presentations of the test stimulus were then interleaved with 6-sec re-adaptation intervals, with zero-contrast gaps of $1.0 \mathrm{sec}$ before and $0.5 \mathrm{sec}$ after each test. Subjects matched the perceived lightness and color of the test by adjusting the luminance and/or chromaticity of a matching stimulus that was presented simultaneously with the test, but in a field placed symmetrically to the other side of the fixation cross. The matching stimulus thus fell on a retinal location that remained adapted to the zero-contrast background. The match stimulus was controlled by a four-button box that varied the match luminance and chromaticity within the plane defining the test and adapting axis. For the luminance vs $L-M$ plane or the luminance vs $S$ plane one pair of buttons varied the match up or down along the luminance axis while the second pair varied the match along the chromatic axis, so that observers could vary perceived lightness and saturation roughly independently. [We discuss our choice of the term "lightness" rather than "brightness" in Webster and Mollon (1993a), though the perception is ambiguous under the conditions of our experiments.] Within the equiluminant plane the button pairs were instead used to vary the angle or distance of the match chromaticity (relative to the background chromaticity), so that perceived hue and saturation could be varied roughly independently. Restricting the match settings to the equiluminant plane rarely resulted in reported failures to achieve a satisfactory match to the equiluminant test stimuli, though within either of the luminancechromatic planes observers often reported that the hue of test stimuli with both luminance and chromatic contrast could be matched only approximately.

On a single run, observers made three separate matches to the same test stimulus, with the match coordinates randomized prior to each setting. The test and re-adaptation sequence continued without interruption throughout the run, and the time required to make the matches was unrestricted. In a daily session subjects matched each of the test stimuli following adaptation to a single adapting axis, with the order of test and adapting stimuli counterbalanced across sessions. The results reported are based on the average of three or six matches from either one or two sessions for each adapting axis. Observers included the authors and four additional subjects, three of whom were unaware of the aims of the experiments.

\section{RESULTS}

\section{Adaptation to chromatic contrast}

Figure 3 shows an example of the matches made to the equiluminant tests following adaptation to modulations along the S (90-270 deg) axis. The outer symbols show the coordinates of the 16 test stimuli, while the inner symbols show the six individual matches made to each test. The r.m.s. deviation of the individual matches from their respective means was 0.93 threshold units. If there were no effect of the adaptation, then the match coordinates should have remained similar to the test coordinates (as confirmed by control measurements without adaptation). Instead, adaptation reduced the perceived contrast of all test stimuli, so that they were matched by less saturated chromaticities that plot closer to the achromatic origin. However, these contrast losses are clearly selective: the largest changes in perceived

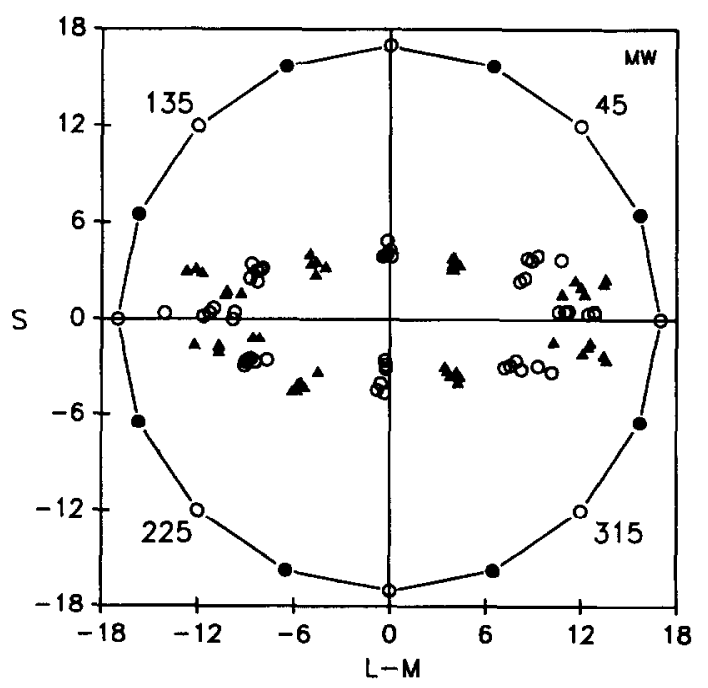

FIGURE 3. Individual matches to test stimuli following adaptation to the S (90-270 deg) axis (observer MW). Outer symbols plot the coordinates of the 16 tests, inner symbols the six matches made to each test. For clarity alternate tests and their corresponding matches are represented by solid or open symbols. 
(A)

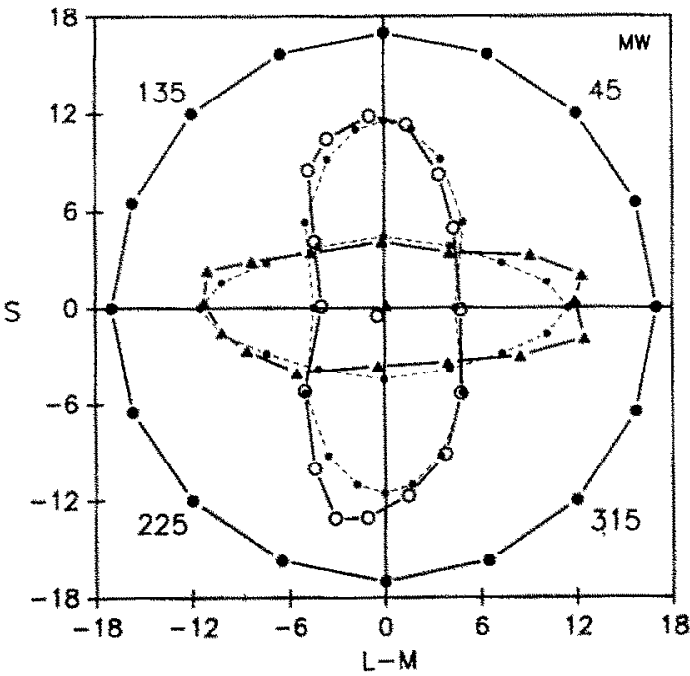

(C)

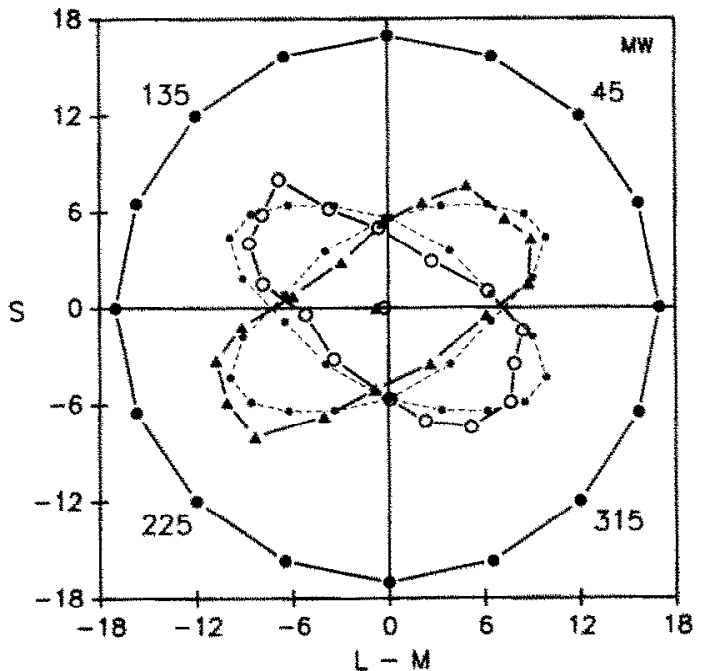

(B)

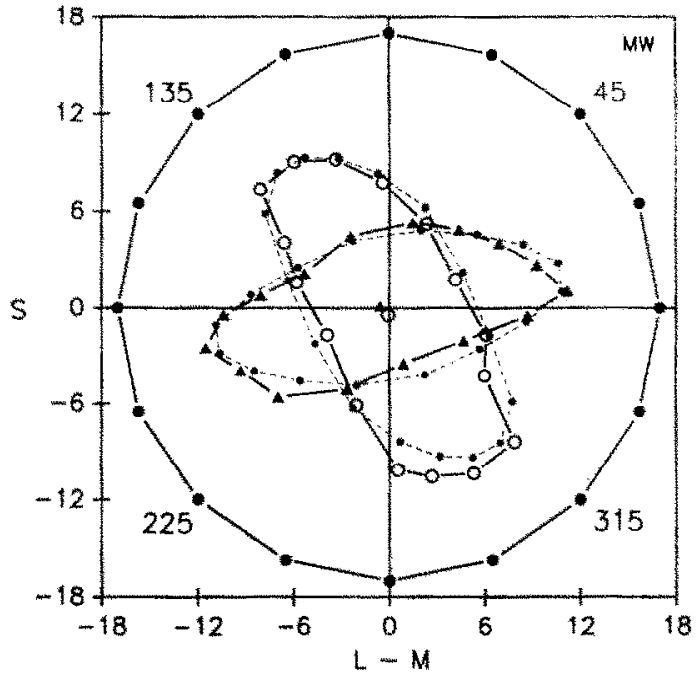

(D)

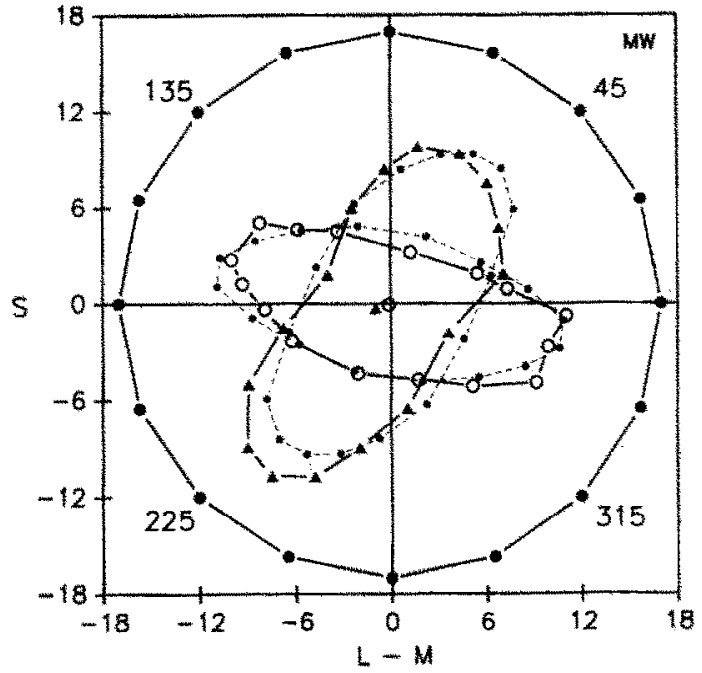

FIGURE 4. Matches to equiluminant test stimuli following adaptation to different directions within the equiluminant plane (observer MW). In each figure the outer solid circles plot the coordinates of the test stimuli, white the inner symbols show the matches to the test stimuli following adaptation to one of two diferent adapting axes that were 90 deg apart. Unconnected symbols near the origin show the mean of the 16 match coordinates. (A) Matches for $L-M(0-180$ deg. circles) or $S$ (90-270 deg, triangles) adapting axes. (B) $22.5-202.5 \mathrm{deg}$ (circles) or 112.5-292.5 deg (triangles) adapting axes. (C) 45 -225 deg (circles) or 135-315 deg (triangles) adapting axes. (D) 67.5-247.5 deg (circles) or 157.5-337.5 deg (triangles) adapting axes, Small circles plot the matches predicted by a model based on multiple chromatic channels (see Discussion).

contrast occur for the tests lying along the $\mathbf{S}$ axis, while the least change was for the tests lying near the $L-M$ axis.

Figure 4 shows for one observer how the perceived color of the test stimuli was affected by adaptation to eight different directions within the equiluminant plane. Each panel plots the mean matches to each test after adaptation to one of two different adapting directions that differed by $90 \mathrm{deg}$. In each case adaptation produced the largest change in perceived contrast for test stimuli that were lying along the axis of the adaptation, while the least loss in contrast was for stimuli that were approx. $90 \mathrm{deg}$ away from the adapting axis. If the channels adapt independently, then a minimum of eight chromatic channels with different spectral sensitivities would be required to account for these aftereffects. These results therefore confirm the variety of chromatic selectivities suggested by changes in detection thresholds following contrast adaptation (Krauskopf et al., 1986b), and suggest that under our suprathreshold conditions the selectivity for chromatic axes intermediate to the $L-M$ and $S$ axes can be very pronounced.

Figure 5 shows similar measurements for two further observers: Observer AS again showed similar selective color changes regardless of the adapting direction. However, for $J M$ the color changes along the $S$ and $L-M$ axes were clearly more selective than for the two intermediate directions tested, with little selectivity evident for the 135-315 deg adapting axis. This result was confirmed for JM by additional trials with this adaptation axis, and is the only case we observed of an apparently non-selective color change. Thus individual 
(A)

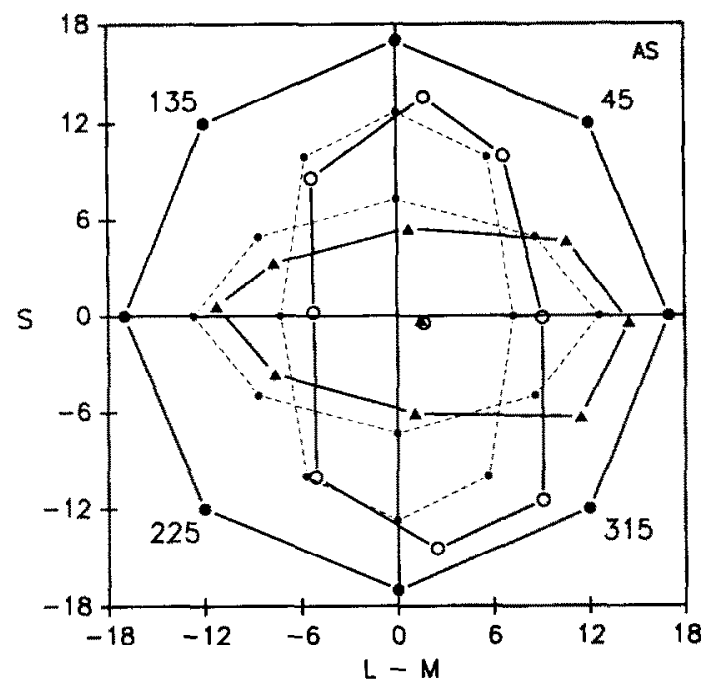

(C)

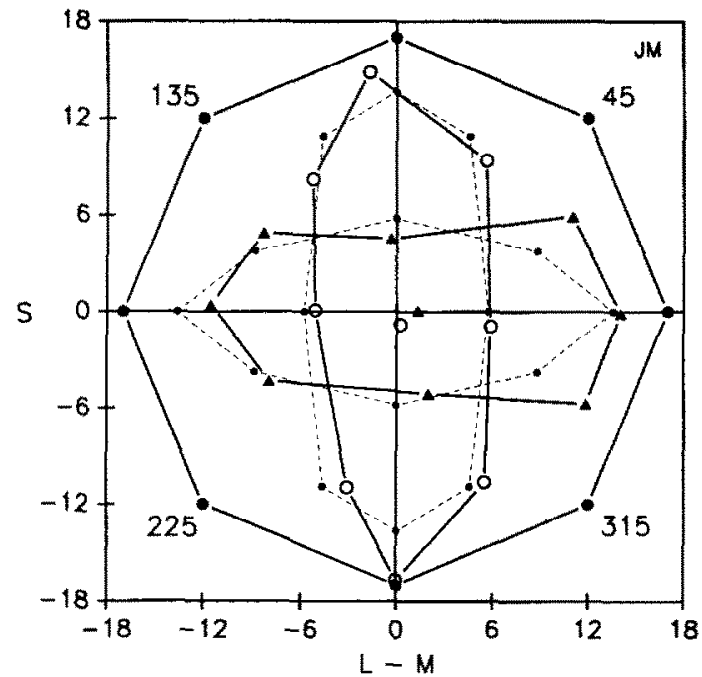

(B)

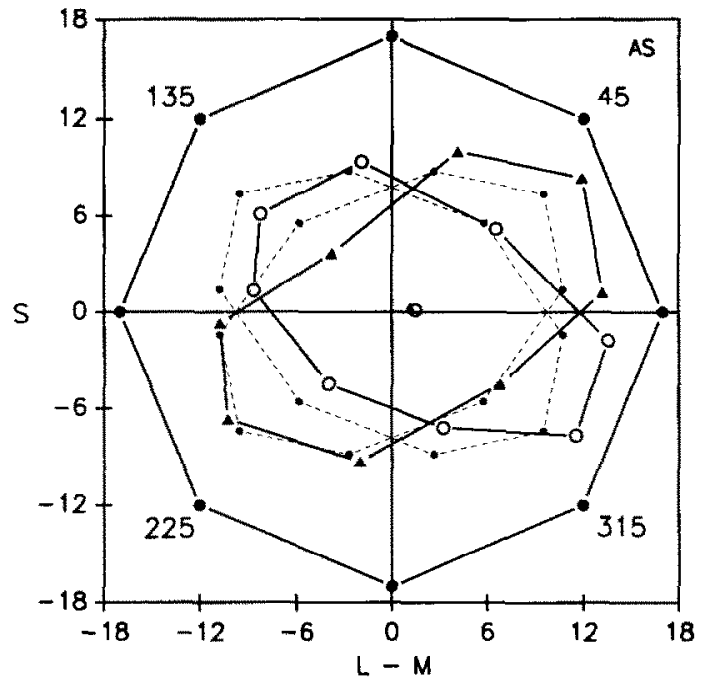

(D)

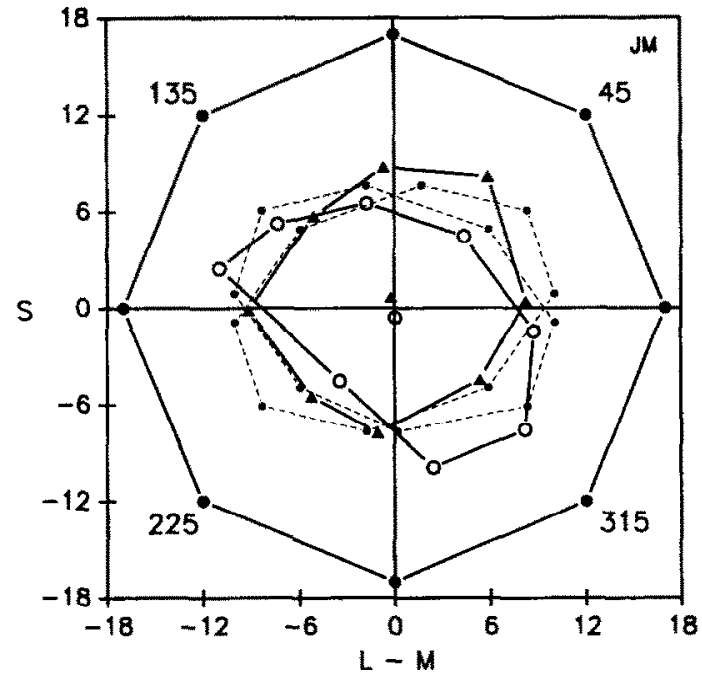

FIGURE 5. Equiluminant matches for two further observers, following adaptation to the $L-M$ or $S$ axes (A and C), or the 45-225 or 135-315 deg axes (B and D). Symbols as in Fig. 4.

differences in these aftereffects are evident among the small sample of observers we have tested.

The large changes in contrast induced by adaptation occurred despite only small changes in the average perceived color of the stimuli, for the mean chromaticity of the 16 match stimuli remained similar to the mean of the 16 test stimuli (as indicated by the unconnected symbols near the origin in Figs 4 and 5). This pattern of aftereffects is very different from the changes that would be expected from adaptation to different mean chromaticities, as in conventional chromatic adaptation (Jameson \& Hurvich, 1972). To confirm this for our

\footnotetext{
*The matches following adaptation to the $+\mathrm{S}$ or $-\mathrm{S}$ mean adapting stimuli are compressed along the $\mathbf{S}$ axis. This is not due to a contrast adaptation effect, but instead results from interposing the $1 \mathrm{sec}$ background chromaticity after the adapting stimulus and before the test stimuli (which was included so that conditions would be identical to our contrast adaptation conditions). When the test stimuli instead immediately followed the mean adapting stimulus there was a roughly constant translation of the perceived test color along a $\log \mathrm{S}$ axis.
}

conditions, we compared for eight of the test stimuli (at 45 deg intervals) the aftereffects of adaptation either to the $\pm 48 \times$ threshold $S$ modulation, or to static adapting colors of $+48 \times$ or $-48 \times$ the $S$ threshold. (Adapting and testing conditions were otherwise identical in these measurements.) Figure 6 shows that adaptation to the different mean colors produced large changes in the mean perceived color (biasing color appearance away from the adapting chromaticity). The absence of these mean changes in the contrast adaptation results thus argues against differences in light adaptation as the basis for the perceived contrast changes.*

\section{Hue changes following adaptation}

Adaptation altered not only the perceived contrast of the test stimuli, but also produced large changes in their perceived hue, or in other words, their perceived direction within the equiluminant plane. In general, these hue changes corresponded to rotations in perceived direction away from the adapting axis, and toward a second, 


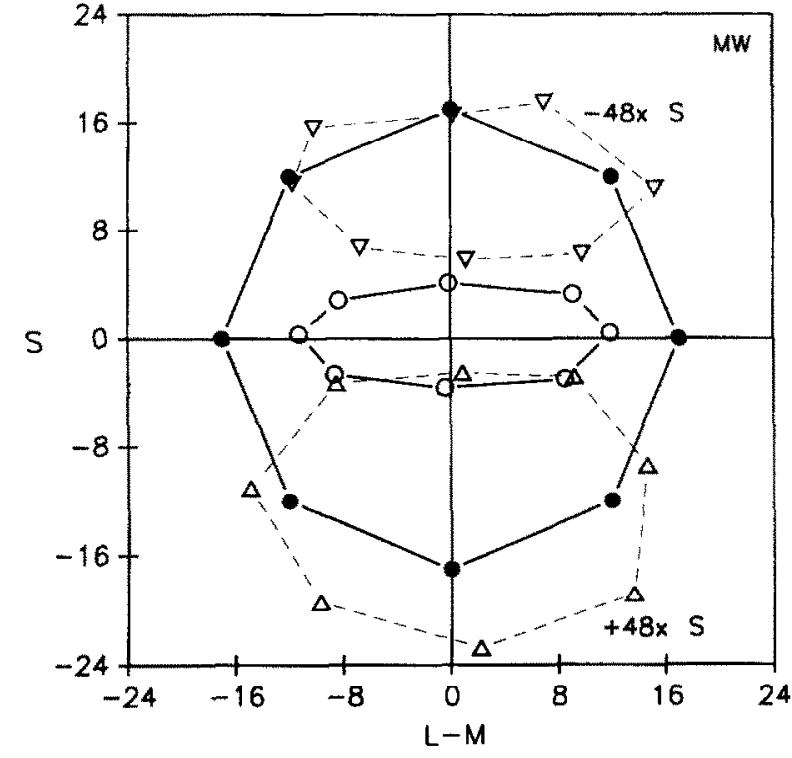

FIGURE 6. Matches following adaptation to a mean change in $\mathrm{S}$ cone signals ( $+48 \times$-threshold or $-48 \times$-threshold, triangles), or to modulations along the $S$ axis of $\pm 48 \times$-threshold (open circles, replotted from Fig. 4). Solid circles plot the coordinates of the test stimuli.

orthogonal axis. In contrast, stimuli lying along either the adapting axis or the orthogonal axis showed little change in perceived hue. That is, stimuli on these two axes might appear to have a lower contrast following adaptation, but they were still matched by a chromaticity that fell along the same direction in color space [a pattern of results analogous to the "displacement paradox" of auditory (von Békésy, 1929) and figural (Osgood \& Heyer, 1952) aftereffects]. Figure 7 shows the changc in perceived direction of the 16 test stimuli following adaptation to modulations along the $L-M$ axis. In this

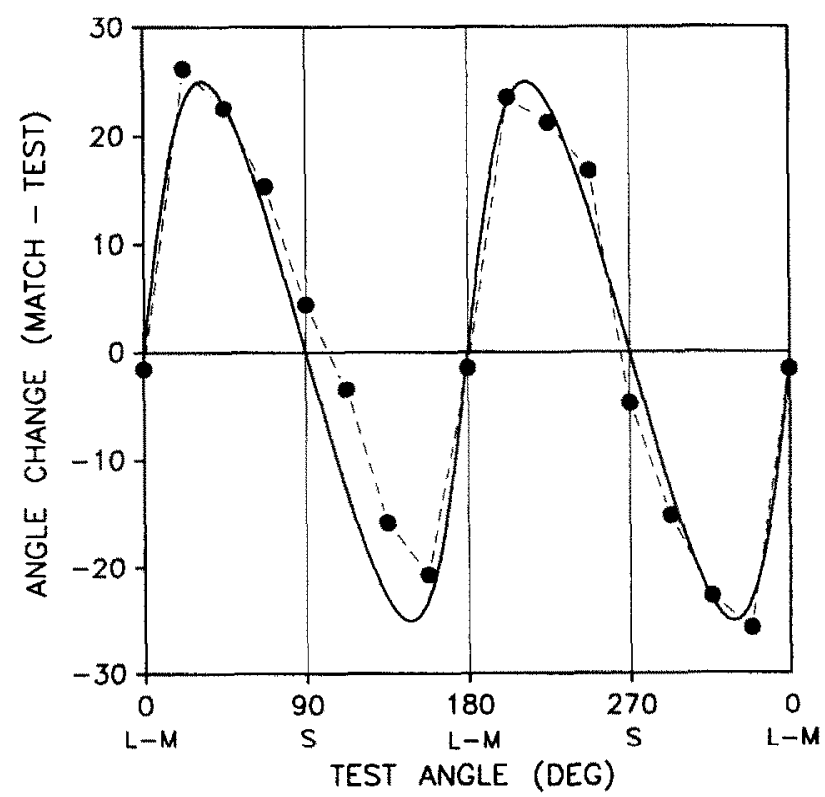

FIGURE 7. Changes in the perceived direction (hue) of test stimuli following adaptation to modulations along the $L-M$ axis. Solid circles plot the difference between the angles of the matching and test coordinates, for each test. Solid line plots the angle changes predicted by the best-fitting multiplicative sensitivity changes along the $L-M$ and $S$ axes (see text). Observer $M W$. case the two tests along either pole of the $L \ldots M$ or the $S$ axes exhibited little change in direction, while the matches to intermediate test angles were rotated away from the $L-M$ axis and toward the $S$ axis. These hue changes could be very large (e.g. $30 \mathrm{deg}$ or more) and pcrceptually very salient.

As we noted in the Introduction, the pattern of hue changes illustrated in Fig. 7 is consistent with differential sensitivity changes in only two chromatic channels selectively tuned to the $L-M$ and $S$ axes. However, if these were the only two chromatic channels, then the hue changes should always rotate relative to these two axes, and the perceived direction of stimuli along the $L-M$ and $S$ axes should remain invariant, no matter what the direction of the adapting axis. We instead found that adaptation to axes intermediate to the $L-M$ and $S$ axes biased the perceived direction of $L-M$ or $S$ tests off their axes. Figure 8 shows the changes in the perceived direction of these tests as a function of the adapting angle for five observers. Large individual differences are evident, yet the general trends in the biases are clear. Note that the biases produced by adaptation had opposite effects in the two test stimuli lying on opposite sides of the $\mathrm{L}-\mathrm{M}$ or $\mathrm{S}$ axes. For example, after adaptation to the $45-225 \mathrm{deg}$ axis (in which $+\mathrm{S}$ and $+\mathrm{L}$ modulations are in phase) the $+\mathrm{L}(-\mathrm{M})$ test required less $S$ cone activity than the background to be matched, while the $+M(-L)$ test required more $S$ cone activity. These opposite changes are again inconsistent with the average change in chromatic sensitivity that would be expected from conventional light adaptation (as illustrated in Fig. 6). Instead, the aftereffects are characterized by rotations of the test axes away from the adapting axis. Similar results were found for all test directions. including stimuli chosen to lie along the unique hue loci. These results therefore suggest that at the level at which the adaptation effects occur, there are no directions within the equiluminant plane that are invariably encoded by only a single chromatic channel.

\section{Hues that do not change following adaptation}

Following adaptation to each single direction in color space, there were two color axes that did not change in perceived hue: one of these directions corresponded to the adapting axis, and was the axis that all other hues were rotated away from, while the second direction corresponded to an axis that all other hues were rotated toward. To examine the relationship between these two directions, we interpolated between the measured hue changes in the test stimuli in order to estimate the four angles that should have remained unaffected by each adaptation axis (i.e. to estimate the four zero-crossings in Fig. 7).

Figure $9($ A) plots these four null angles relative to each of the eight adaptation directions for observer $\mathrm{MW}$. If there were a fixed direction in color space whose angle remained invariant, then the points would plot along a line of slope -1 in this figure. Instead, the null directions vary consistently with the adapting direction. For each adaptation axis two of the null angles (near 0 and 
(A)

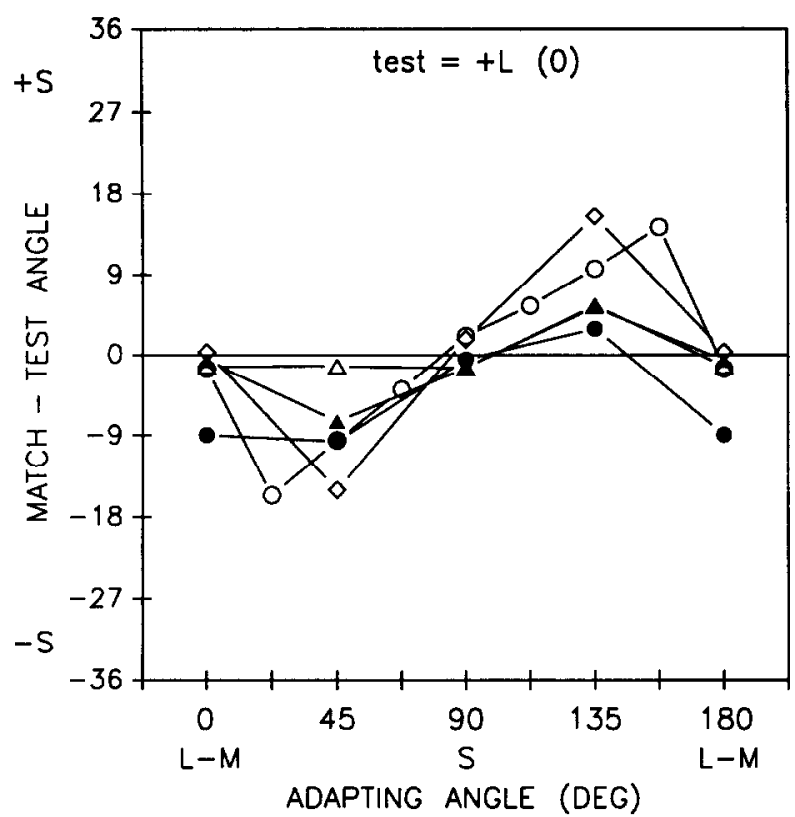

(C)

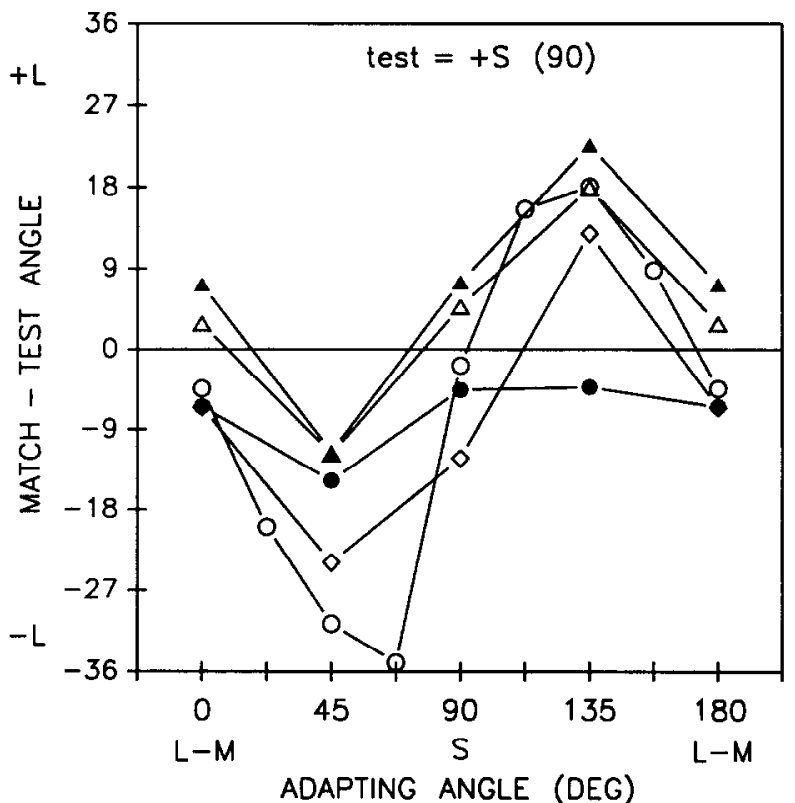

(B)

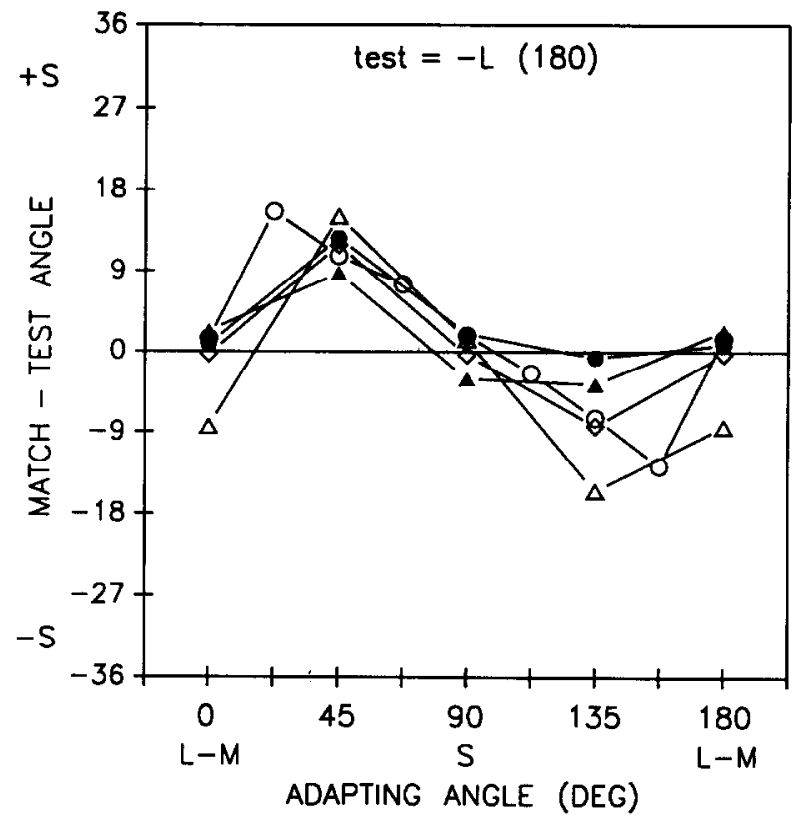

(D)

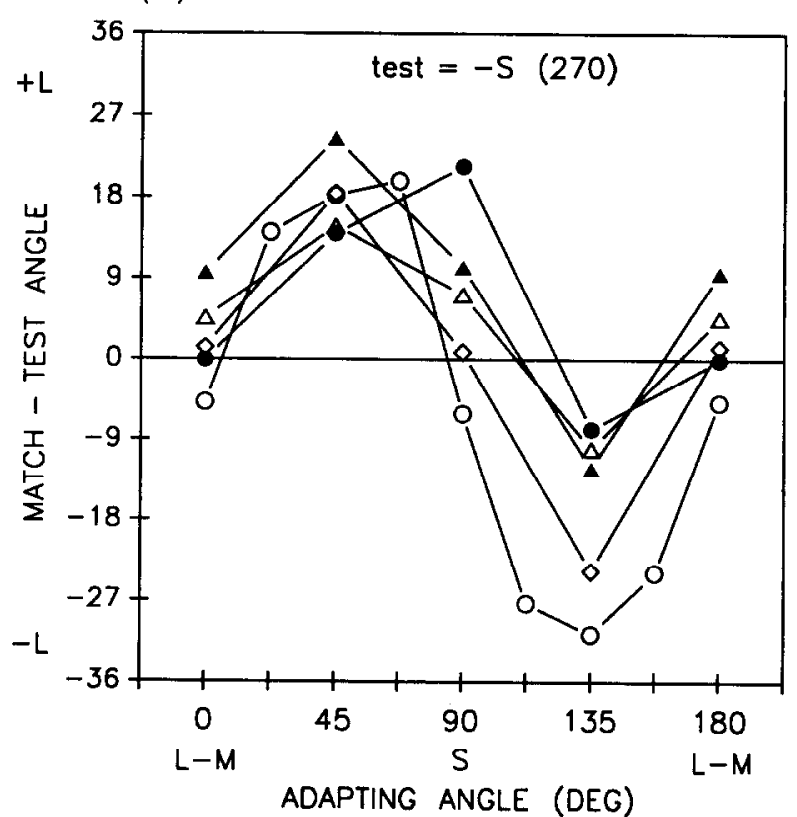

FIGURE 8. Changes in the perceived direction of the four test stimuli that differed from the background only in $\mathrm{L}-\mathrm{M}$ contrast or only in $S$ contrast, following adaptation to different directions within the $S$ and $L-M$ plane. (A) $+L(-M)$ test. (B) $-\mathrm{L}(+\mathrm{M})$ test. (C) $+\mathrm{S}$ test. (D) $-\mathrm{S}$ test. In each figure the difference between the angles of the match and test coordinates are plotted as a function of the angle of the adapting stimulus for five observers (MW, open circles; JM, solid circles; $\mathrm{AE}$, open triangles; AS, solid triangles; JP, open diamonds). In (A) and (B), positive angle changes indicate that the $L-M$ test was matched by a chromaticity that produced more $S$ cone excitation than the background. In (C) and (D), positive angle changes indicate that the $S$ test was matched by a chromaticity that produced more $L$ (less $M$ ) cone excitation than the background.

$180 \mathrm{deg})$ lie close to the adapting axis. The second pair of angles define approximately an axis that is on average $90 \mathrm{deg}$ from the adapting axis, yet systematic deviations are evident whenever the adapting axis was intermediate to the $S$ or $L-M$ axes. Such deviations could arise if our original threshold scaling of contrasts along the $S$ and $\mathrm{L}-\mathrm{M}$ axes were inappropriate (either because the thresholds were in error or because a different scaling applied above threshold). For example, Fig. 9(B) illus- trates how changing the relative weights assumed for the $S$ and $L-M$ axes will affect the apparent angle between two vectors. Increasing the $S$ to $L-M$ ratio by some factor, $\alpha$, changes the original angles, $\Theta_{1}$, by

$$
\Theta_{2}=\tan ^{-1}\left[\alpha \tan \left(\Theta_{1}\right)\right] .
$$

Note that this stretching does not affect the direction of stimuli lying along either the $S$ or the $L-M$ axes, yet for any intermediate pair of axes there is some rescaling 
that will render the pair $90 \mathrm{deg}$ apart. We therefore assessed whether there is a single rescaling of the $\mathrm{L}-\mathrm{M}$ and $S$ axes that would leave all of the null axes $90 \mathrm{deg}$ from the adapting axes. This was done by finding the $S$ to $\mathrm{L}-\mathrm{M}$ contrast ratio that minimized the sum of squared differences between the measured null angles and the predicted nulls (corresponding to the adaptation axis $\pm 90 \mathrm{deg}$ ).

Figure $9(\mathrm{C})$ replots the null angles after rescaling the original threshold ratio of $\mathrm{S}$ to $\mathrm{L}-\mathrm{M}$ by 1.49 . With this revised scaling the null angles for all adapting directions are close to 90 deg apart, with an r.m.s. error of 6.9 deg. This scaling thus provides a measure of the relative strengths of the $S$ and $L-M$ cone signals in our specific task, and suggests that our nominal circles of test and adapting chromaticities are in fact ellipses elongated along the $\mathrm{S}$ axis. In terms of cone contrasts it suggests that a contrast of 0.01 in the $S$ cones was equivalent to opposing $\mathrm{L}-\mathrm{M}$ contrasts of 0.0016 in the $\mathrm{M}$ cones and 0.0008 in the $\mathrm{L}$ cones.

(A)

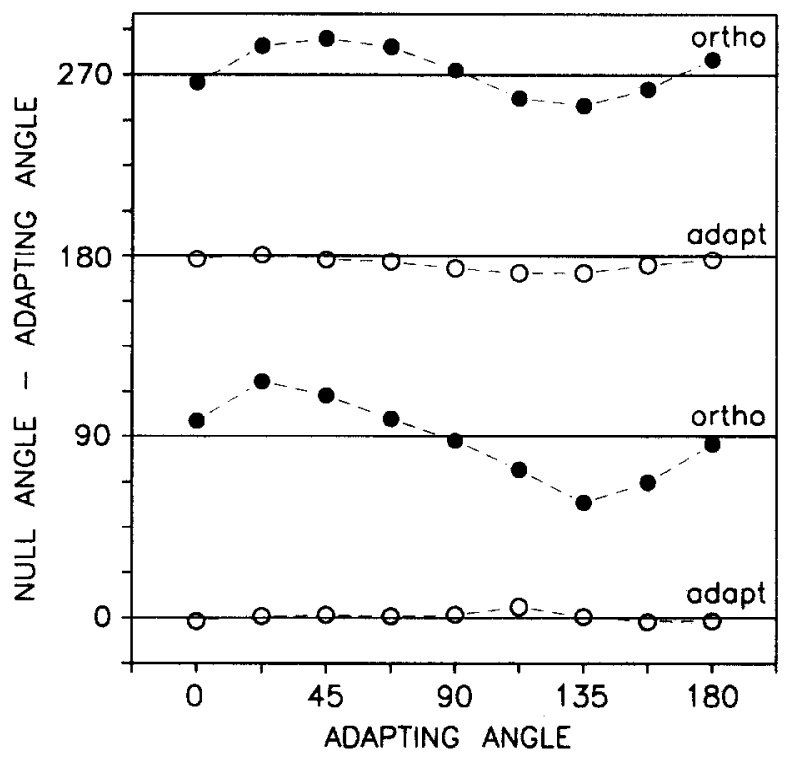

(B)

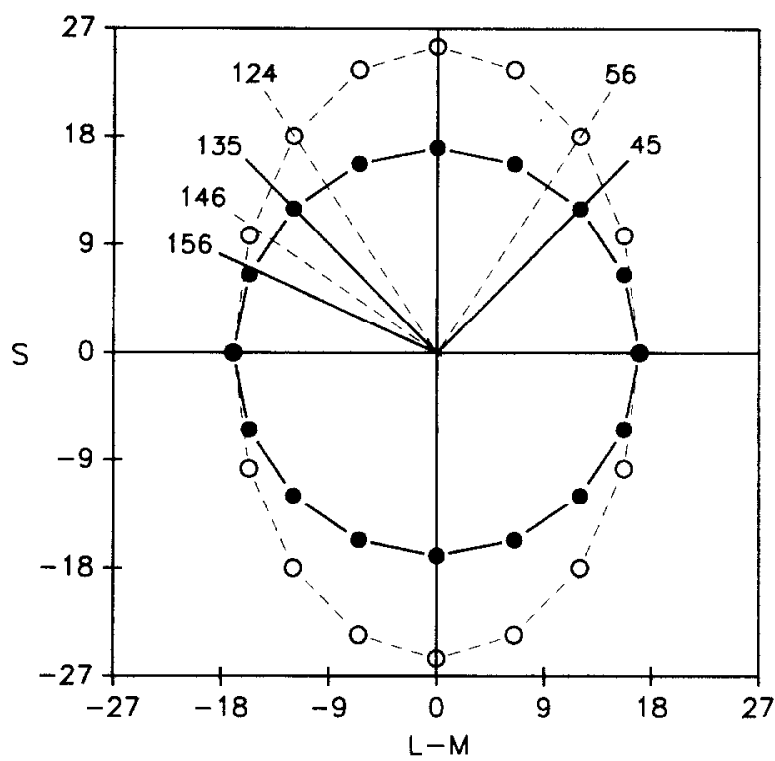

(C)

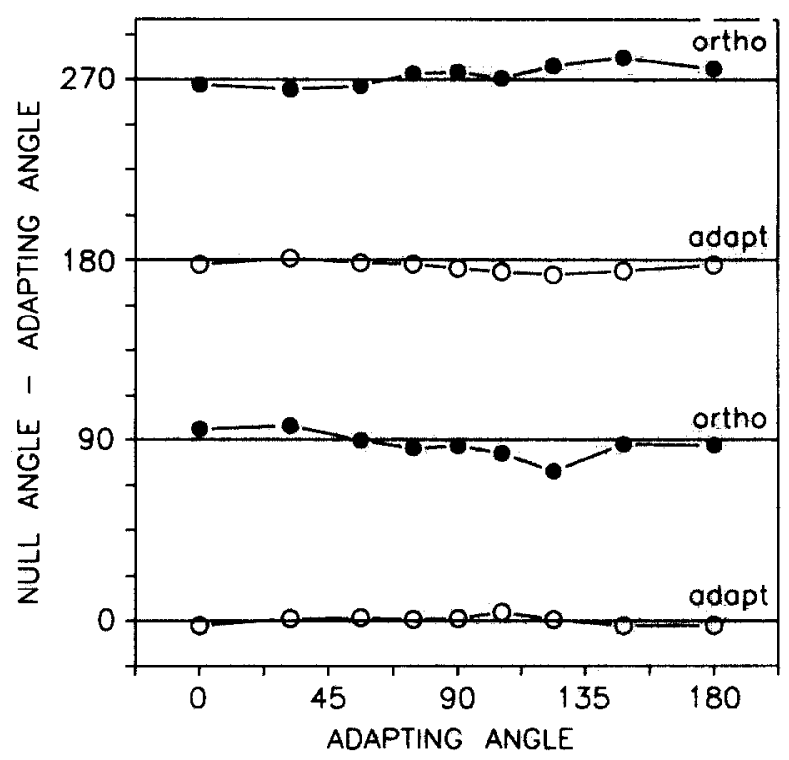

FIGURE 9. (A) Estimates of the test axes that do not change in perceived direction for each adapting direction. Open circles represent opposite poles of an axis that all other directions are biased away from. Solid circles estimate the two poles of the axis toward which all hues rotate. The angles are plotted relative to each adapting angle. Thus ordinates of " 0 deg" and " 90 deg" correspond to each adapting angle and its orthogonal angle, respectively. (B) Coordinates of test stimuli plotted either in the original threshold-scaled space (solid circles), or assuming $\mathrm{S}$ signals are $1.5 \times$ stronger (open circles). The revised scaling rotates all oblique angles toward the $S$ axis. For example, the nominal angles of 45 and $135 \mathrm{deg}$ are transformed to 56 and $124 \mathrm{deg}$. respectively, and thus are no longer orthogonal. The axis orthogonal to $56 \mathrm{deg}$ is instead $146 \mathrm{deg}$, which in the original scaling corresponds to an angle of $156 \mathrm{deg}$ (and thus is $156-45=111 \mathrm{deg}$ from the nominal $45 \mathrm{deg}$ axis). (C) Null angles of (A) replotted after rescaling $\mathrm{S}$ contrasts by 1.49 . This scaling was chosen to minimize the deviation of each solid circle from the axis orthogonal to the adapting axis. 
The simple structure of the rescaled null angles suggests that the direction of hue changes in any test can be closely predicted from the angular difference between the test and adapting directions within the appropriately weighted $S$ and $L-M$ plane. The $S$ and $L-M$ dimensions represent orthogonal linear combinations of the cone excitations. The observed hue changes are thus consistent with sensitivity changes in channels whose chromatic selectivities depend on different linear combinations of cone signals. However, at least for some observers, the hue changes provide little evidence for any bias toward a preferred axis within this representation, a bias that might be expected if the sensitivities of the channels were strongly biased along specific color directions.

\section{Relationship of hue changes to unique hues}

The organization of chromatic signals along independent $S$ and $L-M$ dimensions can be contrasted with a representation in which signals corresponding to the red-green and blue-yellow loci of phenomenal color appearance are encoded independently. Krauskopf $e t a l$. (1982) showed that threshold elevations following adaptation to a unique blue-yellow axis were less selective than the sensitivity changes following adaptation to the $\mathrm{L}-\mathrm{M}$ or $\mathrm{S}$ axes, indicating that the channels underlying the threshold changes were more clearly tuned to the cardinal directions than to the unique blue-yellow axis. As Figs 4 and 5 show, these differences in selectivity were not always apparent in our suprathreshold measures. Nevertheless, we could evaluate these alternative models because they predicted different patterns of hue change in the test stimuli.

Figure 10(A) plots the locations of the four unique hues (red, blue, green, and yellow) within our equiluminant plane (rescaled by our revised estimate of the relative sensitivities along the $\mathrm{S}$ and $\mathrm{L}-\mathrm{M}$ axes). The angle corresponding to each hue was estimated at two different contrasts $(17 \times$ and $48 \times$ the original contrast threshold) by varying the color angle of the $0.5 \mathrm{sec}$ test in four randomly interleaved staircases, with the observer required to judge on each trial the color deviation from the unique hue (e.g. "too red" or "too green" for unique yellow). Consistent with previous measures (e.g. Burns, Elsner, Pokorny \& Smith, 1984), the red-green axis lay close to the $\mathrm{L}-\mathrm{M}$ axis but was curved, while blue and yellow were colinear along an axis intermediate to the two cardinal directions.

If unique blue-yellow and unique red-green were encoded independently, then adaptation to unique blue-yellow should have no effect on the perceived hue of unique red or unique green. On the other hand, the hue changes illustrated in Fig. 9 suggest instead that blue-yellow adaptation should cause all hues to rotate toward an axis $90 \mathrm{deg}$ away from the blue-yellow axis within the appropriately weighted $L-M$ and $S$ plane. To test this, we adapted to modulations along the blue-yellow axis and then matched the appearance of a range of test stimuli near unique red or unique green. Figure 10 plots the change in perceived angle of test directions spanning either the green [Fig. 10(B)] or the red [Fig. 10(C)] color angles. Also shown are the null directions predicted by the orthogonal directions. Unique green showed little change following adaptation, but it was close to $90 \mathrm{deg}$ from the blue-yellow axis based on our revised scaling of the $S$ and $L-M$ axes, and this result is thus consistent with either model. On the other hand, a clear hue change was found for unique red, which shifted in appearance toward blue, and the color angle that did not change was again close to the angle predicted by the orthogonal axis in the $S$ vs $L-M$ plane.

\section{Contrast response changes following adaptation}

As an index of the selectivity of the adaptation effects for different adapting directions, we compared the sensitivity changes along the adapting and orthogonal axes for each adaptation axis. This analysis required us to assess the functional form of the response changes produced by color contrast adaptation, or in other words, to assess how contrast adaptation alters the contrast response function. To estimate directly the functional form of the sensitivity changes, we measured the effects of the $48 \times$-threshold adapting modulations on a wide range of test contrasts. Figure 11 plots the changes in perceived contrast along the adapting and orthogonal axes for adapting and test axes that were either along the $\mathrm{L}-\mathrm{M}$ or $\mathrm{S}$ axes, or along axes of 45-225 or 135-315 deg based on our revised $(1.49 \times)$ scaling of $\mathrm{S}$ contrasts. These intermediate axes therefore corresponded to angles of 56.1-236.1 and 123.9-303.9 deg in our original threshold scaling. (Again this rescaling does not alter the angles of the cardinal axes.) Symbols show the log change in perceived contrast as a function of the test contrast. Over the full range of contrasts the perceived contrast changes were larger along the adapting axis than along the orthogonal axis, indicating a selective aftereffect for each adapting direction. In fact, in these measurements the response changes for intermediate or cardinal axes were largely indistinguishable. At suprathreshold contrasts the stimuli could be matched for both saturation and hue. Hue angles for the matching stimuli remained similar to the test angles, for tests on both the adapting and orthogonal axes. This suggests that the scaling of weights along the cardinal axes is largely independent of test contrast.

Figure 11 also shows the contrast changes predicted by the best fitting multiplicative or subtractive changes in the perceived contrast of the test stimuli. A multiplicative adaptation effect predicts that any test contrast will be reduced by the same proportion, and thus plots as a constant log change in the figure. Alternatively, a subtractive adaptation effect predicts that any test will be reduced in contrast by the same absolute amount. The observed contrast changes are intermediate to the predictions of these two models. Both can be shown to be inadequate descriptions of the contrast changes by assessing the changes in perceived contrast by one-way ANOVAS: Significant effects of test contrast were found whether the contrast changes were expressed as the 
(A)

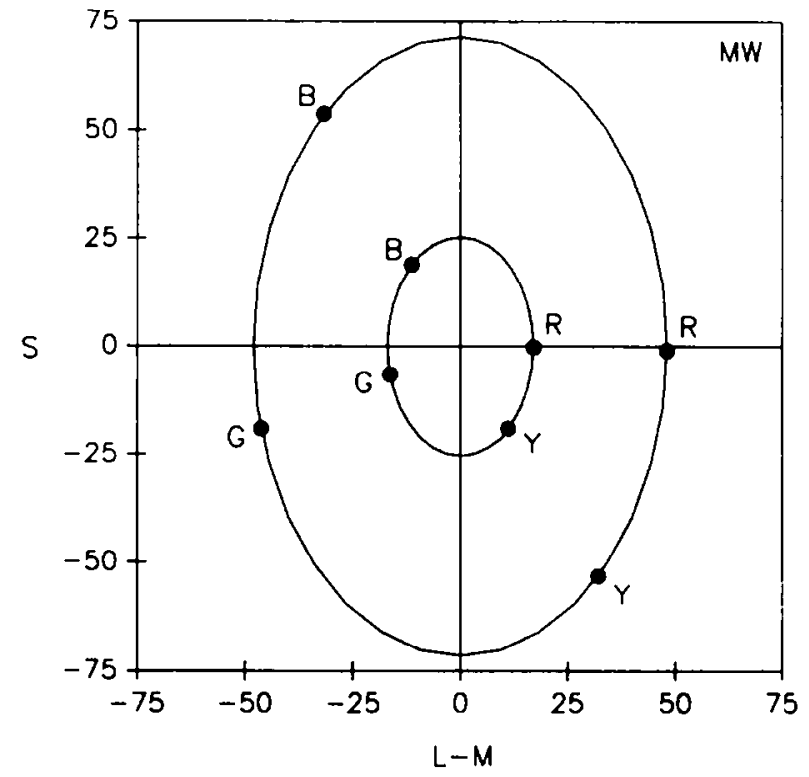

(B)

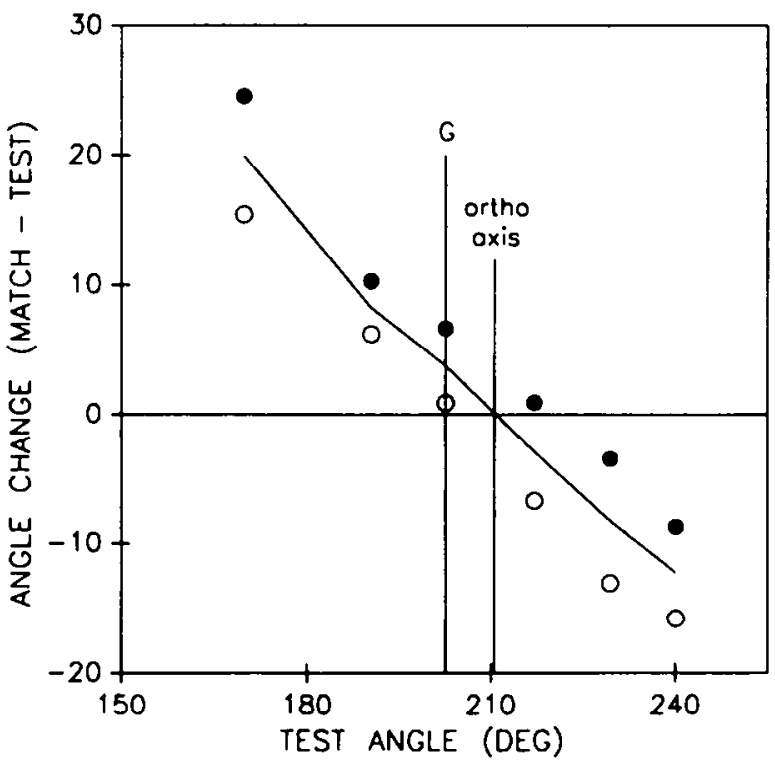

(C)

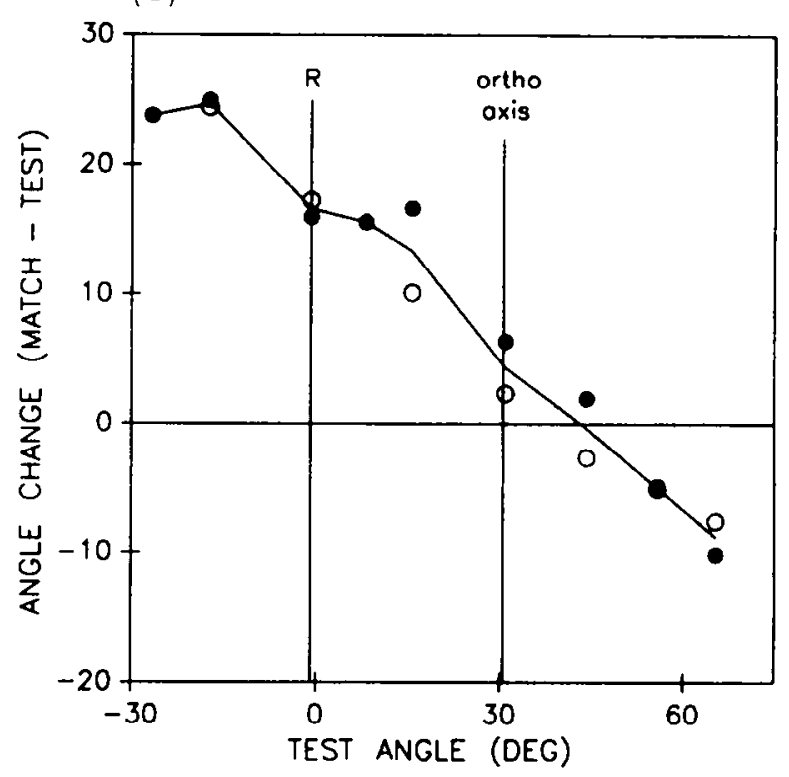

FIGURE 10. (A) Location of the unique hues within the rescaled equiluminant plane. The angle corresponding to each unicuc hue is shown at two different contrasts. (B) Changes in the perceived direction of test angles spanning the pre-adapt unique-green axis, following adaptation to the unique bluc yellow axis. Solid line shows the mean of measurements from two different sessions (unconnected symbols. based on three matches each). Orthogonal axis is the angle 90 deg from the blue -yellow adapting axis. (C) Changes in the perceived direction of test angles spanning the pre-adapt unique-red axis.

linear (subtractive) or the $\log$ (multiplicative) difference between the match and test stimuli. Instead, the observed contrast changes approximate power functions, similar

*These functions describe the perceived contrast changes only for the single adaptation contrast and the range of test contrasts we measured, for adaptation typically has little effect on test contrasts higher than the adapting contrast (Blakemore et al., 1971: Georgeson, 1985), while the power functions instead predict increases in the perceived contrast of high contrast tests. Additional measurements at a reduced $L-M$ adapting contrast of $24 x$. threshold produced little change in $48 \times$-threshold tests while producing large changes in test contrasts below $24 \times$-threshold. These equations would also fail to predict differences in selectivity of the adaptation effects for different adapting axes. as were evident for some observers to the aftereffects reported by Blakemore et al. (1971) for contrast changes in luminance sinewave gratings. Exponents were similar for the different adapting axes. For results pooled across different adapting axes, the changes in perceived contrast $(\Delta C)$, as a function of test contrast $\left(C_{\mathrm{t}}\right)$ were best fitted by

$\Delta C=-1.36 C_{1}^{0.64}$ for tests on the adapting axis. and $\Delta C=-0.26 C_{1}^{0.12}$ for tests on the orthogonal axis.*

Figure 12 shows the matches made following adaptation to the $\mathrm{L}-\mathrm{M}$ axis for observer $\mathrm{MW}$ (replotted from Fig. 4) and compares them with the sensitivity changes predicted by the multiplicative or subtractive 


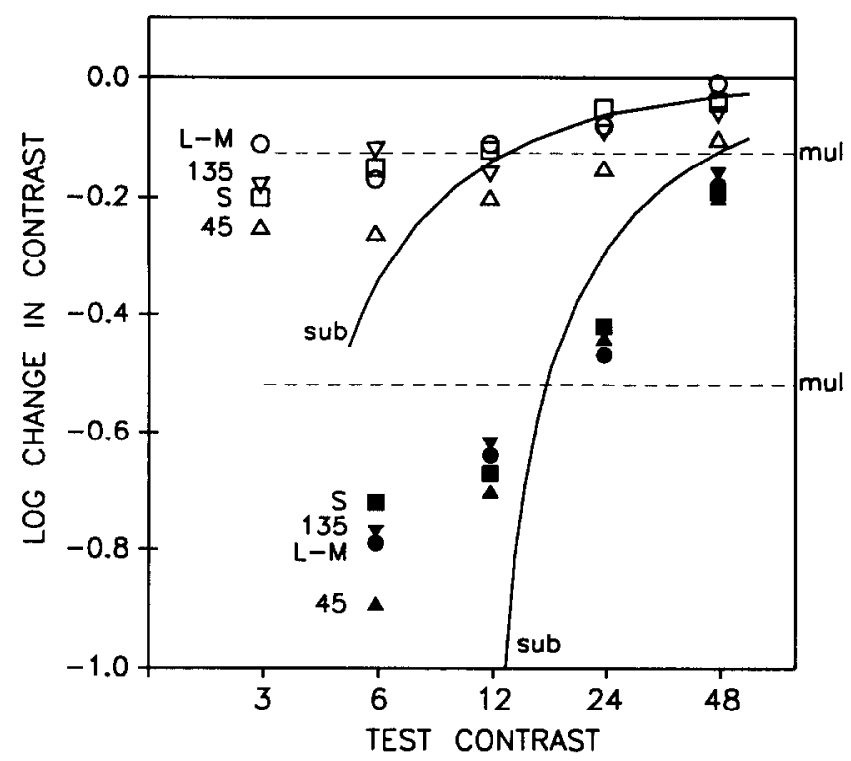

FIGIJRE 11. Fffects of $48 \times$ adapting contrasts on tests of different contrasts. The log change in matching contrast is plotted for tests lying along either the adapting axis (solid symbols) or the orthogonal axis (open symbols). The different symbols plot the results for the four different adapting axes tested (indicated for the data points for the matches to lowest test contrasts), and are the average of 12 settings (six each from tests on opposite poles of the axis). Solid lines show the best-fitting constant subtractive change in test contrast along either the adapting or orthogonal axes, while dashed lines plot the best-fitting multiplicative change.

models or the empirically defined power function. The resulting matches are reasonably approximated by either the power function or the multiplicative change. On the other hand, subtractive changes provide a poor description of the matches: the absolute loss in perceived contrast along the adapting axis is too large to account for the absolute change at intermediate test angles. Differences between the models are also evident in the predicted hue changes in the test stimuli. A subtractive effect exhibits large deviations from the observed changes in perceived test direction, yet these hue changes are well fitted by either the multiplicative (plotted in Fig. 7) or the empirical power function. These differences were clear across all adaptation angles and for all

\footnotetext{
*For adapting angles intermediate to the $S$ and $L-M$ axes, this analysis initially failed to account for the observed color changes, because it often predicted sets of matches that exhibited the wrong orientation. For example, it is apparent in Fig. 4(C) that the matches following adaptation to the $45-225$ or $135-315$ deg axes form ellipses whose orientations are biased toward the $L-M$ axis. These biases could again arise from an inappropriate scaling of the signals along the $S$ and $L-M$ axes. Thus as a second measure of this scaling, we varied the nominal $S$ to $L-M$ contrast ratio to find the single value that gave the best fit of the observed matches for all oblique adapting axes. For observer $\mathrm{MW}$, this required increasing the relative weights assumed for $\mathrm{S}$ signals by a factor of 1.37 , close to the value of 1.49 estimated from the perceived hue changes. Similar scaling constants were required to fit the matches for three further observers, though a fifth observer's matches were instead consistent with the original scaling. Note that while this rescaling markedly improved the fits to the matches, it had very little effect on our estimates of the multiplicative sensitivity changes, when these changes were expressed as the log loss in sensitivity.
}

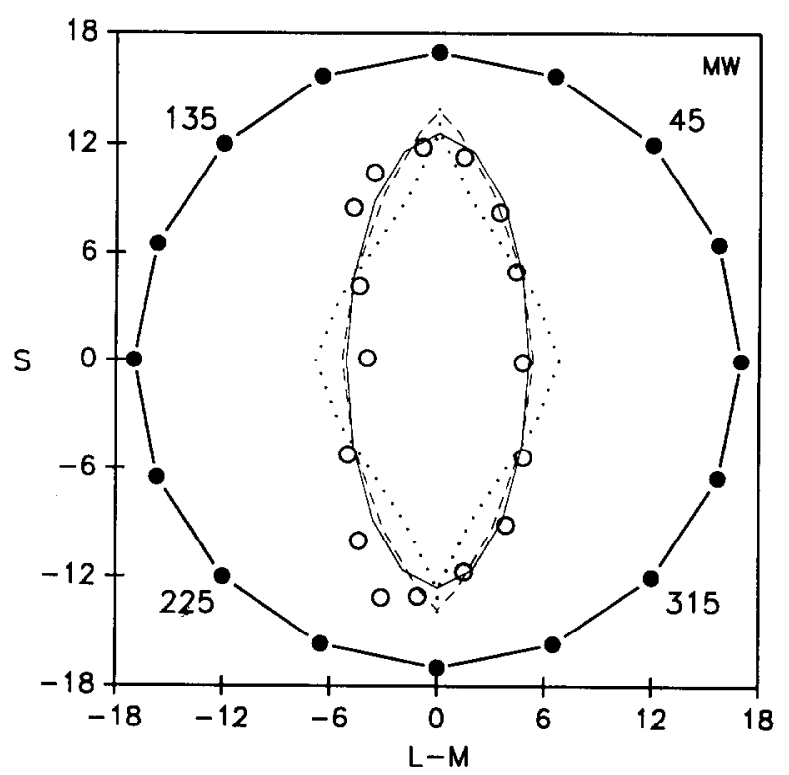

FIGURE 12. Matches following adaptation to the $L-M$ axis (open circles, replotted from Fig. 4), compared to a multiplicative (solid line) or subtractive (dotted line) adaptation effect, or to the empirically defined power function (dashed line). The functions were fitted by assuming independent sensitivity changes in the components of the test vector along the adapting ( $L-M)$ and orthogonal (S) axis. For the multiplicative or subtractive models, the magnitudes of constant log or linear changes along the two axes were varied to minimize the sum of square deviations from the 16 match coordinates. The predictions for the power function were instead constrained by equation (3). Differences between the means of the 16 match and 16 test coordinates were subtracted out prior to fitting the functions, though this had little effect on the calculated sensitivity changes.

observers. Thus for the restricted range of contrasts examined in matches to the $17 \times$-threshold tests, a multiplicative change appeared to provide a close and simple approximation to the observed adaptation effects. We therefore used a multiplicative sensitivity change in order to compare the selectivity for different adapting directions. However, the form of the contrast changes exhibited in Fig. 12 may not relate directly to how adaptation is changing the responses in individual channels, for it also depends on how selective the channels are for different color directions, and if there are more than two chromatic channels, it will depend on how the responses across channels are pooled (see Discussion).*

Figure 13 compares the predicted multiplicative sensitivity changes along the adapting and orthogonal axes for the different adapting directions. With only one exception for one observer (JM, 135-315 deg adapt) the estimated sensitivity changes are larger along the adapting axis than along the orthogonal axis, again indicating that the adaptation effect can be selective for any direction within the equiluminant plane. However, for all but one observer (AS) aftereffects were assessed by this procedure to be more selective following adaptation to the cardinal axes than to intermediate directions. This analysis therefore confirms the special status of these chromatic directions in contrast adaptation (Krauskopf et al., 1982), but again suggests that comparable selectivity also occurs for intermediate adapting axes for some observers. For all observers the effects of adaptation on 


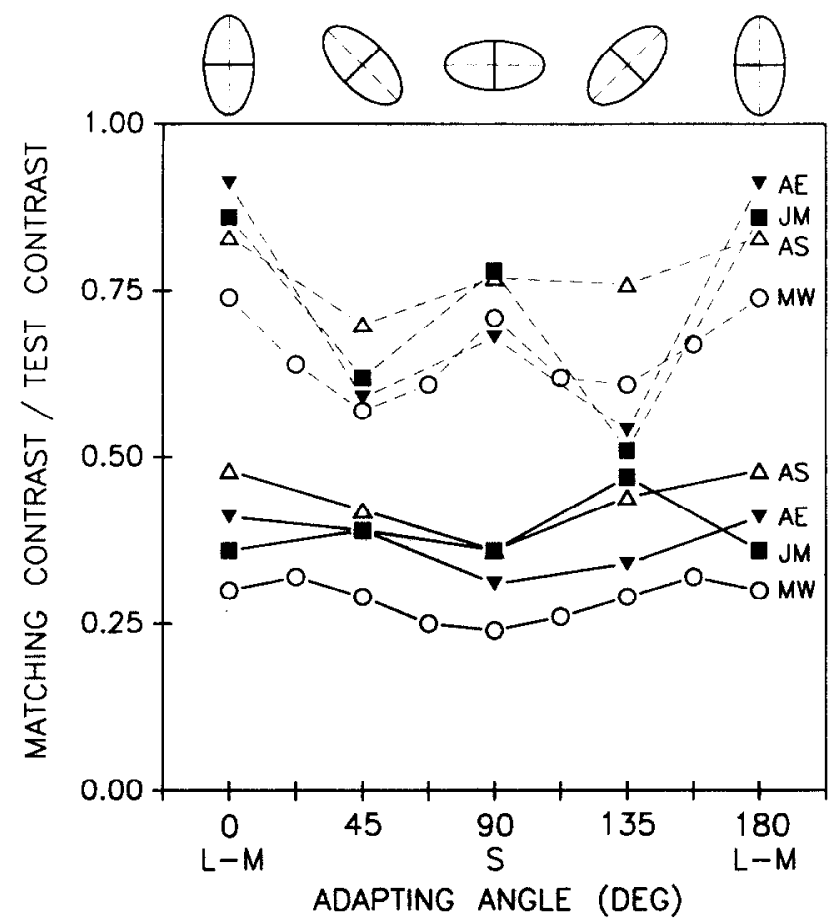

FIGURE 13. Predicted multiplicative sensitivity changes along adapting (solid lines) and orthogonal (dashed lines) axes for different adapting directions. Individual curves correspond to the different observers tested.

the perceived color of any test is in general well described by the independent sensitivity changes that adaptation produces along the adapting and orthogonal axes. This suggests that the changes in perceived contrast and perceived hue of the test stimuli reflect the same underlying response changes.

\section{Adaptation to combined luminance and chromatic vari- ations}

In this section we consider how adaptation to stimuli that vary in chromaticity and/or luminance affect both the perceived color and the perceived lightness of test stimuli. Figures 14 and 15 show for three observers the matches made to test stimuli following adaptation to different directions within the plane defined by the luminance and $\mathrm{L}-\mathrm{M}$ chromatic axes. Changes in the perceived color and lightness of the test stimuli were again selective for the direction in color-luminance space of the adapting modulation. That is, the largest changes in perceived contrast corresponded to the direction of the adapting axis. This suggests that the appearance of the stimuli depends on adaptable mechanisms that can be selectively tuned to

*No differences were evident among the matches made following adaptation to +90 or $-90 \mathrm{deg}$ phase shifts between the luminance and chromatic variations. Thus, at least as assessed by our $0.5 \mathrm{sec}$ pulsed tests, the aftereffects do not appear to be selective for the angular direction (clockwise vs counter-clockwise) of the luminance-color change. We also did not observe differences between the perceived color changes in our pulsed tests following sawtooth vs sinusoidal modulations along a single axis, though sawtooth modulation has been found to elevate thresholds differentially for step changes along different poles of the adapting axis (Krauskopf et al., 1982). different combinations of luminance and chromatic contrast. These selective interactions between suprathreshold luminance and chromatic stimuli were observed even when the chromatic variations modulated activity only in the $S$ cones (Fig. 16), even though the $S$ cones appear to make little contribution to conventional measures of luminance sensitivity (Lennie et al., 1993).

Within the luminance and $\mathrm{L}-\mathrm{M}$ plane, adapting angles of 45-225 or $135315 \mathrm{deg}$ correspond to roughly equal modulations in luminance and $+\mathrm{I}(-\mathrm{M})$ chromaticity that are added together either in phase (45-225 deg) or $180 \mathrm{deg}$ out of phase (135-315 deg). However, in both cases the temporal variations in luminance and chromaticity are correlated. We also tested the effects of adaptation when the same luminance and chromatic components were shifted in phase by $90 \mathrm{deg}$, so that luminance and chromaticity were not linearly correlated. This results in an adapting stimulus that varies along a circle of fixed radius [ $(48 / \sqrt{ } 2) \times$-threshold], in the luminance-chromatic plane, rather than along a single axis. The matches following adaptation to this uncorrelated color-luminance variation are plotted along with the 45-225 and 135-315 deg results in Fig. 14(C), and reflect the largely nonspecific sensitivity losses that would be expected for all three of these adapting stimuli if the channels were sensitive only to the independent luminance and chromatic variations in the stimuli. The fact that the same luminance and chromatic components instead produced different adaptation effects, depending on how they were combined with each other, again indicates that there are adaptable mechanisms that can be selectively tuned to these covariations.*

To compare how selective the aftereffects were for different adapting directions, we again approximated the matches by fitting independent multiplicative sensitivity changes along the adapting and orthogonal axes. These estimates confirmed that the adaptation effect was selective for any direction tested. All observers tended to show more selective changes following equiluminant chromatic adaptation than following luminance adaptation, which surprisingly, appeared little more selective than intermediate adapting directions. This asymmetry between luminance and chromatic adaptation reflects in part the finding that chromatic adaptation had less effect on the perceived contrast of luminance tests than vice versa. Chromatic adaptation and luminance tests were in fact the only conditions that consistently suggested a lack of cross adaptation between any adapting and test axes.

Adaptation to different luminance/chromatic axes again induced systematic distortions in the perceived direction of the test stimuli, with no test direction remaining invariant. As an example, Fig. 17 plots for four observers the change in perceived direction of the two equiluminant $\mathrm{L}-\mathrm{M}$ test stimuli or the two pure luminance test stimuli as a function of the direction of the adapting axis. Note again that these changes were rotations of the test axes away from the adapting axis, and thus adaptation induced opposite effects on opposite poles of the test axes. For example, after adaptation to the 45-225 deg axis (a modulation between "bright-red" and 
(A)

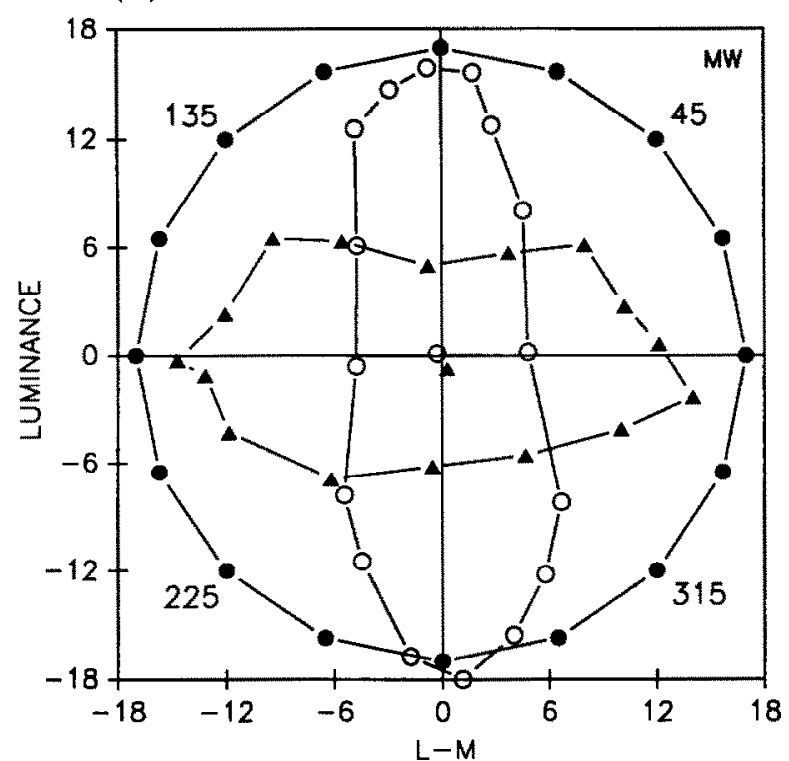

(C)

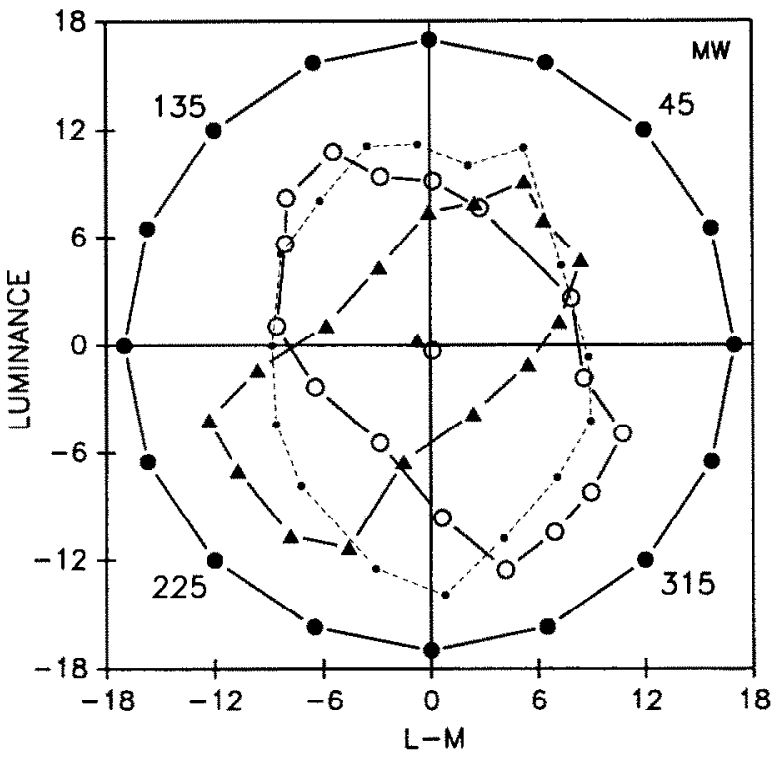

(B)

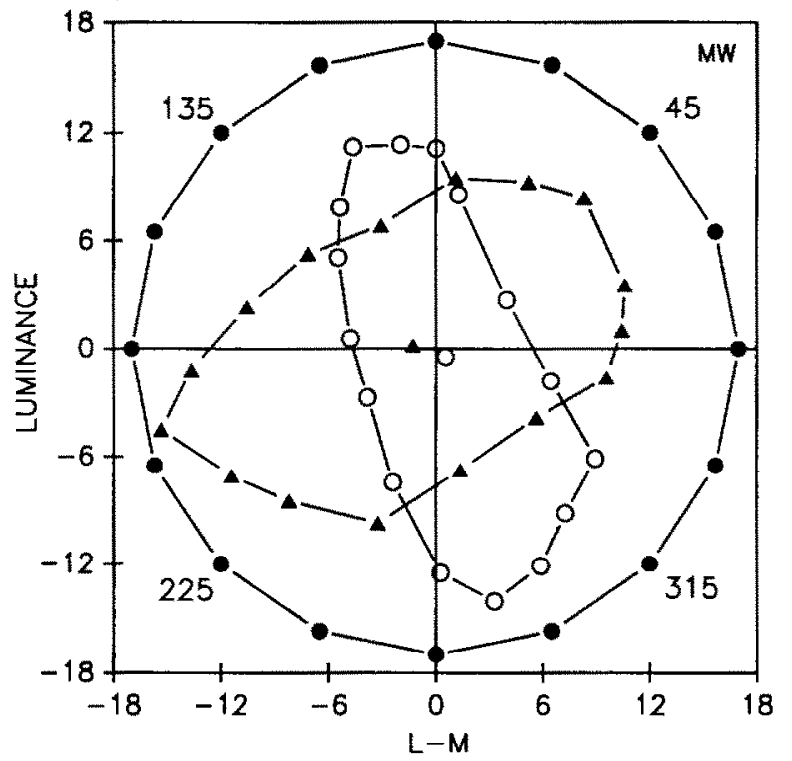

(D)

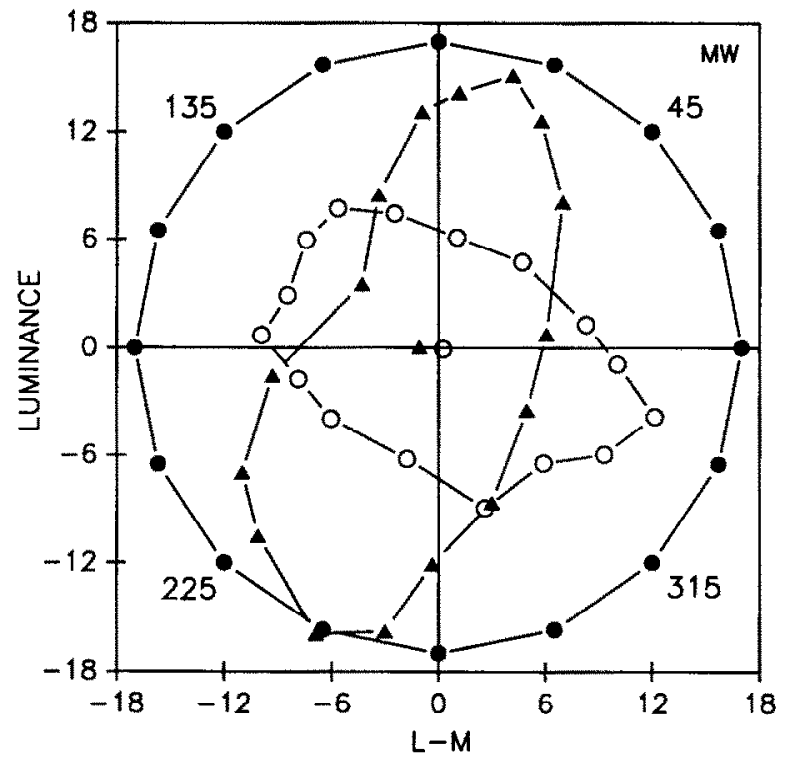

FIGURE 14. Matches to test stimuli following adaptation to different directions within the luminance and $L-M$ plane, for observer MW. Symbols as defined in Fig. 4. (A) Matches following adaptation to pure chromatic (L - M, circles) or pure luminance (triangles) adaptation. (B) 22.5-202.5 deg (circles) or 112.5-292.5 deg (triangles) adapting axes. (C) 45-225 deg (circles) or 135-315 deg (triangles) adapting axes. (D) 67.5-247.5 deg (circles) or 157.5-337.5 deg (triangles) adapting axes. (C) Also shows the matches following adaptation to uncorrelated luminance and $\mathrm{L}-\mathrm{M}$ chromatic contrast (small solid circles), achieved by modulating luminance and chromaticity $90 \mathrm{deg}$ out of phase in the adapting stimulus. The luminance and chromatic components were the same as for the 45-225 and 135-315 deg adapting stimuli, in which luminance and $+\mathrm{L}(-\mathrm{M})$ chromaticity instead vary in phase (45-225 deg) or $180 \mathrm{deg}$ out of phase (135-315 deg).

"dark-green") the $+\mathrm{L}(-\mathrm{M})$ chromatic test (equiluminant "red") appeared darker than the background, while the $-\mathrm{L}(+\mathrm{M})$ chromatic test (equiluminant "green") appeared brighter than the background. Similarly, adaptation to this axis caused the pure luminance increment to appear greencr, while the pure luminance decrement appeared redder. These aftereffects suggest that pure luminance or pure chromatic stimuli are not uniquely encoded by purely luminance-sensitive or purely chromatic-sensitive mechanisms. Similar aftereffects occurred for all of the test directions. Figure 18 plots estimates of the test angles that did not change in perceived direction for each adapting axis (estimated for observer MW as in Fig. 9). Again it is evident that these null angles do not correspond to fixed directions in color space, but instead vary systematically with the adapting direction. To a first approximation, the perceived hue and lightness of any test rotates away from the adapting axis and toward the orthogonal axis. However, null angles for intermediate adapting axes (i.e. for modulations in both luminance and chromaticity) appeared systematically shifted off the adapting axis and toward the chromatic axis (so that test stimuli on the adapting axis were rotated in appearance toward the luminance 
axis). This suggests that intermediate adapting axes produced differentially larger changes in chromatic sensitivity than in luminance sensitivity. This result is in contrast to the null angles determined for the equiluminant plane (which instead remained close to the adapting axis), and cannot be compensated by rescaling the axes. Instead, it again suggests an asymmetry between the adaptation effects for luminance and chromatic contrast.

\section{Effects of adaptation on reaction times}

As an alternative, performance, measure of the contrast changes following adaptation, we measured the effects of adaptation on reaction times for detecting the test stimuli, under the assumption that lower perceived contrasts should take longer to respond to (Henmon, 1906). For these measurements the screen was divided into four 2 deg fields symmetric about the fixation point and separated by 0.4 deg gaps. The adapting stimulus was presented in all four quadrants in phase. After a $0.5 \mathrm{sec}$ delay, the test stimulus was displayed in one of the four fields until the observer identified the quadrant by pressing one of four buttons. In each session observcrs adapted to a single adapting axis and were then tested for four randomly interleaved test stimuli (corresponding to the two poles of the adapting or orthogonal axis).

Figure 19 shows the reaction times for stimuli within the equiluminant plane. The reaction times are plotted as a function of test contrast before or after adaptation either to the $\mathrm{L}-\mathrm{M}$ or $\mathrm{S}$ axes, or to intermediate directions of $45-225$ or $135-315 \mathrm{deg}$. Reaction times were similar for the different test directions. and asymptoted with increasing test contrast. Following adaptation to each adapting direction, test stimuli that were along the axis of adaptation took substantially longer to
(A)

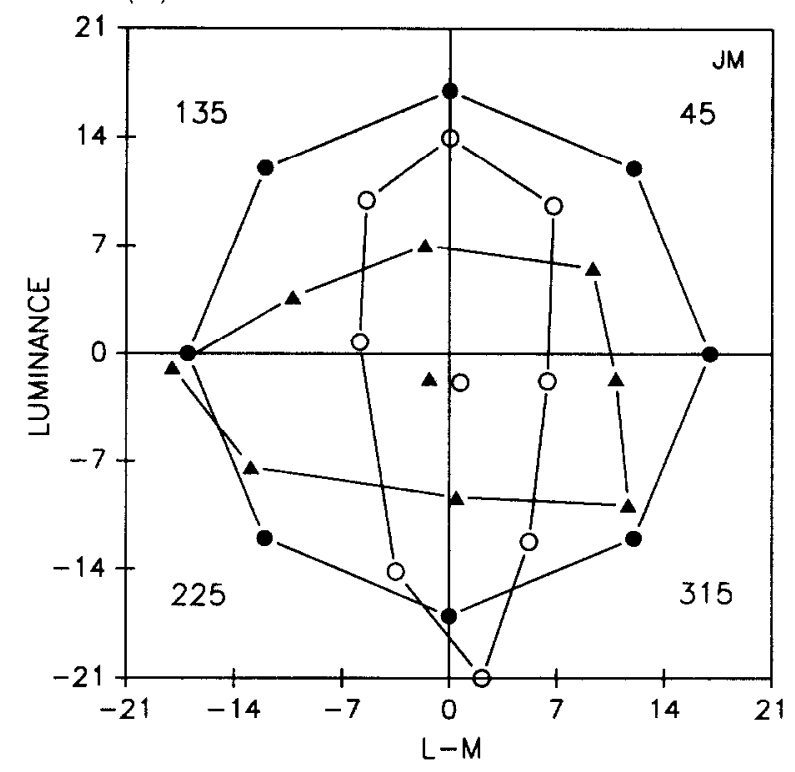

(C)

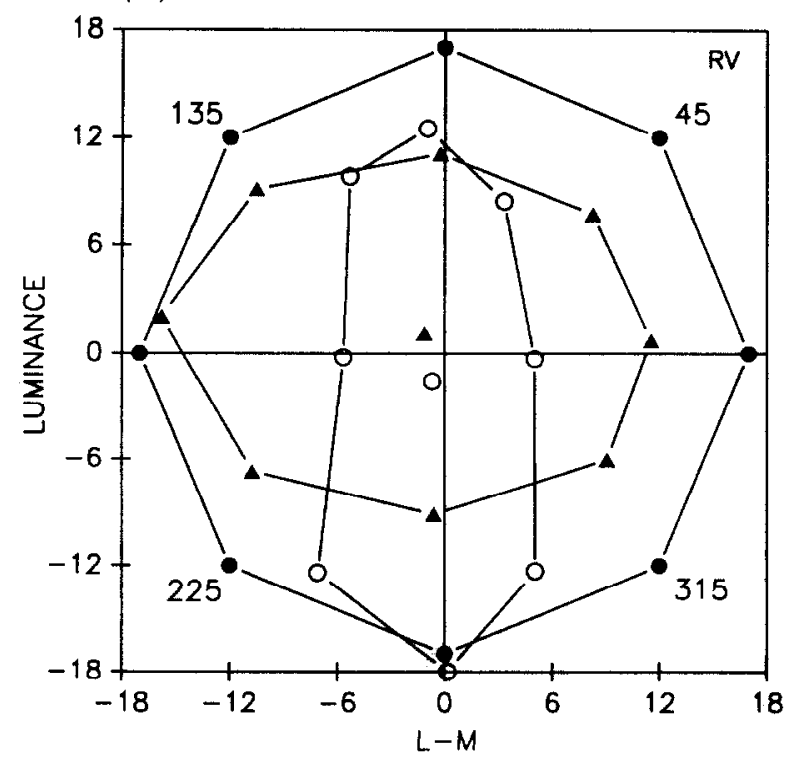

(B)

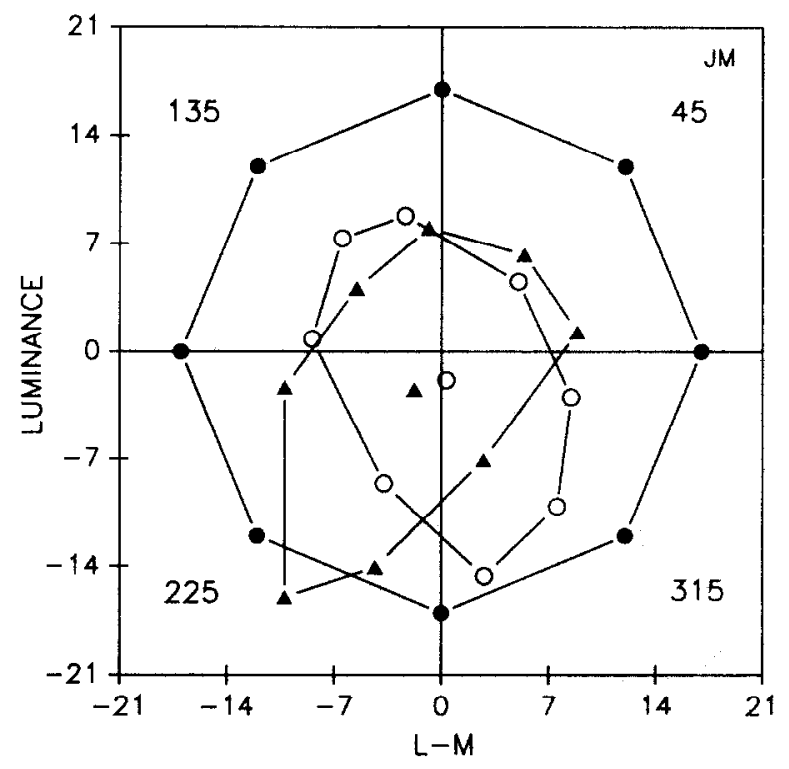

(D)

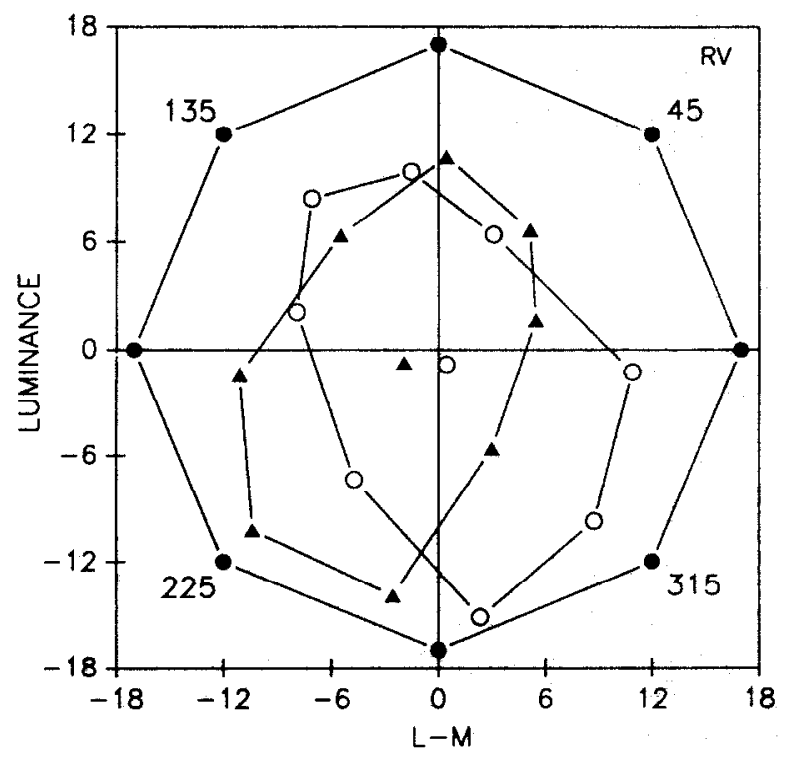

FIGURE 15. Matches for two further observers, following adaptation to the $\mathrm{L}-\mathrm{M}$ or luminance axes (A and C), or the $45-225$ or $135-315 \mathrm{deg}$ axes (B and D). Symbols as in Fig. 4 
(A)

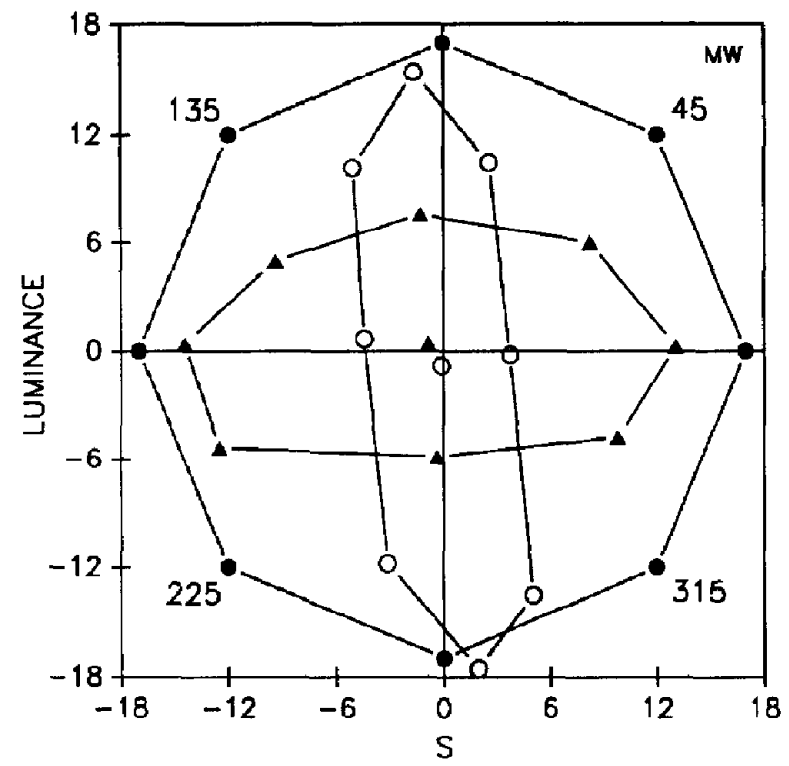

(B)

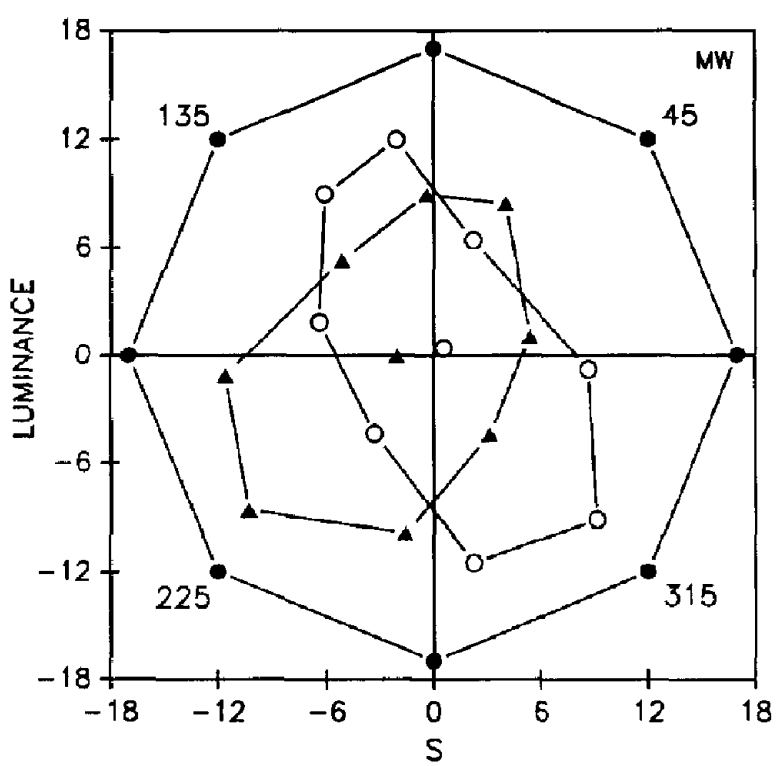

(D)

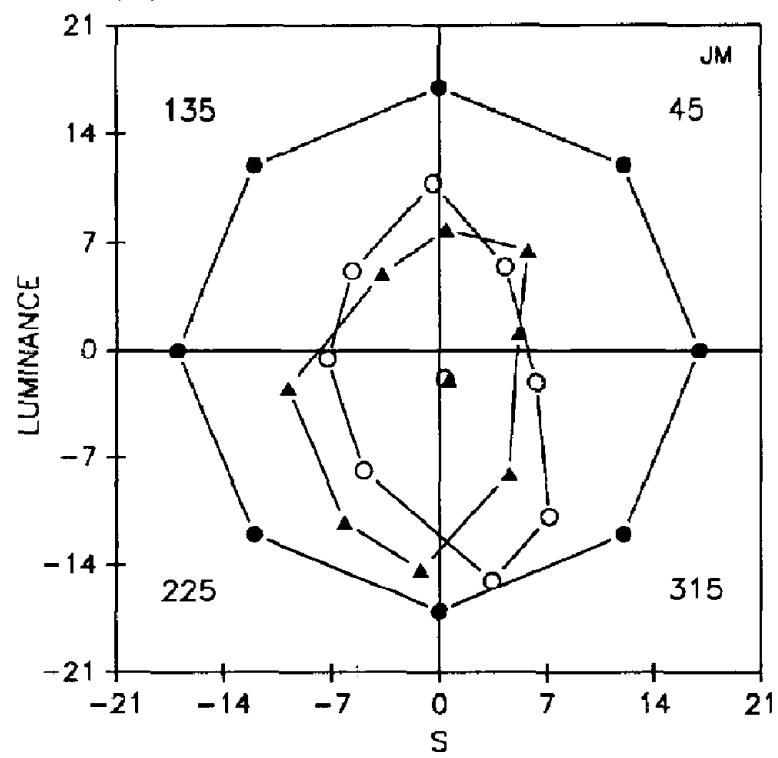

(C)

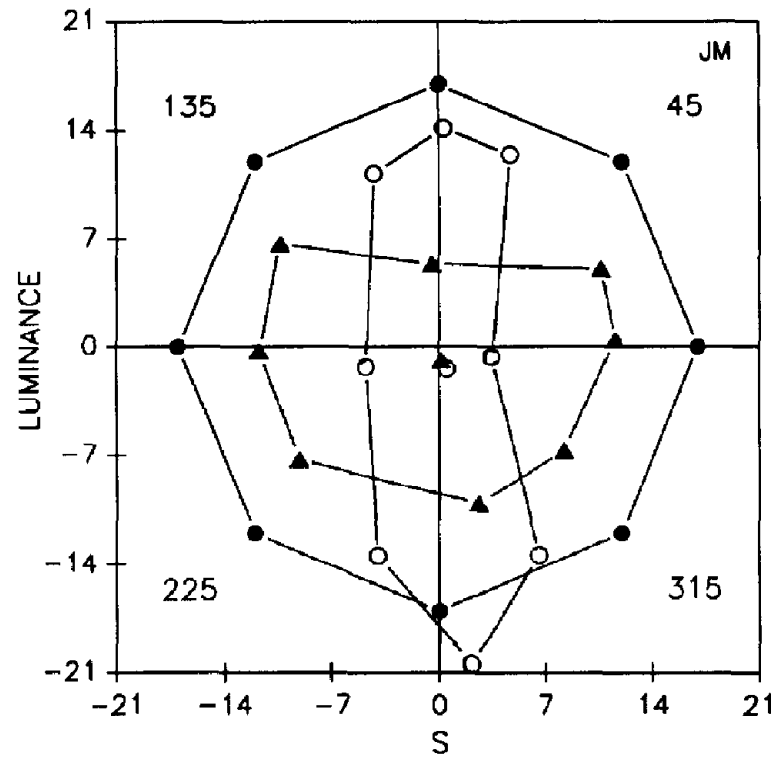

(E)

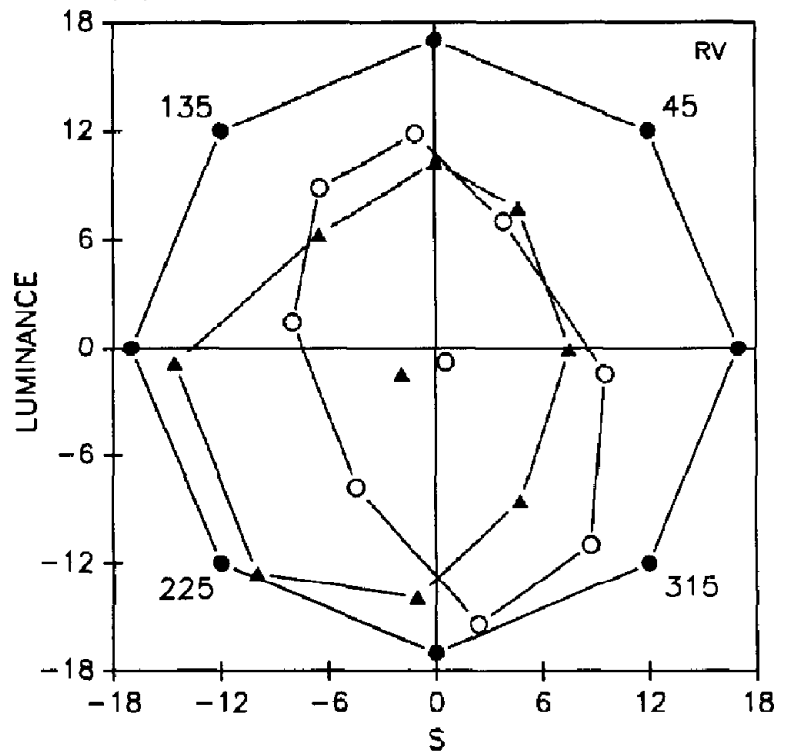

FIGURE 16. Matches for two observers following adaptation to the luminance or $S$ axes (A and C), or the 45-225 or 135-315 deg axes (B and D). (E) Matches for a third observer tested only for the combined luminance and $S$ adaptation. 
respond to. Thus these results are consistent with the selective changes in perceived contrast measured by our matching procedure, and suggest that these selective changes are also evident at very low test contrasts. Figure 20 shows similar results for observer JM. Surprisingly his reaction times indicate clear selectivity for the 135-315 deg adapting axis, though this selectivity was not evident in his matching results (Fig. 5).

Figures 21 and 22 show how adaptation affected reaction times to stimuli within the two luminance- chronatic planes. (In these measurements the delay between the adapting and test interval was increased to $1.0 \mathrm{sec}$, and this longer delay accounts for the weaker adaptation effect in the chromatic stimuli compared to Fig. 19.) Reaction times for luminance tests were consistently shorter than for chromatic tests, with both again asymptoting at low contrasts. Adaptation again showed significant selectivity for the combined-luminance and chromatic axes (as assessed by Mann-Whitney tests of the reaction times for tests lying along the test axes vs
(A)

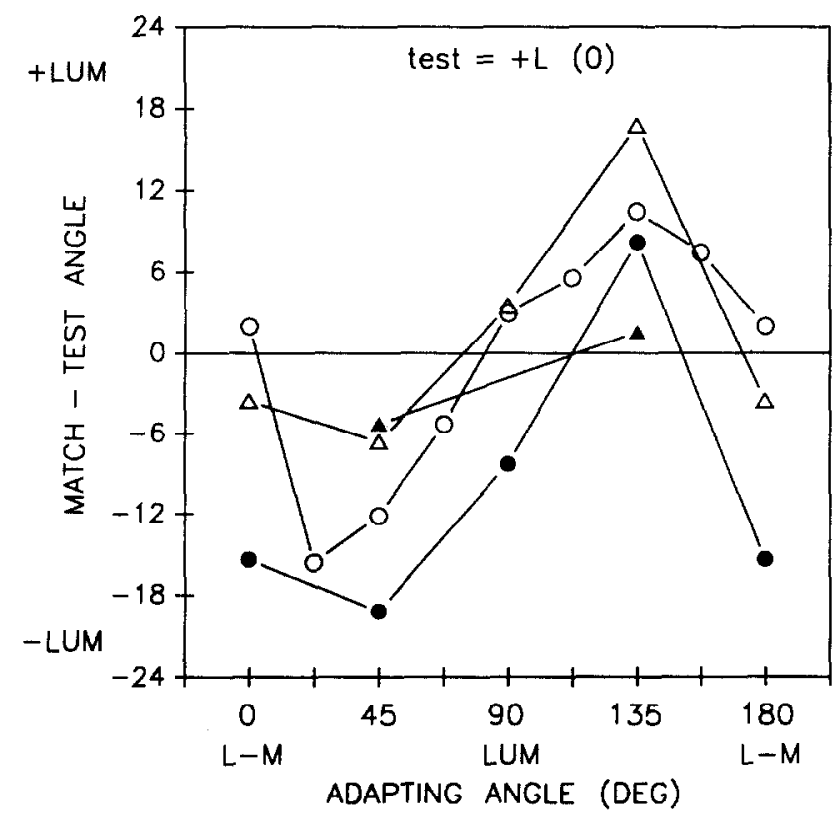

(C)

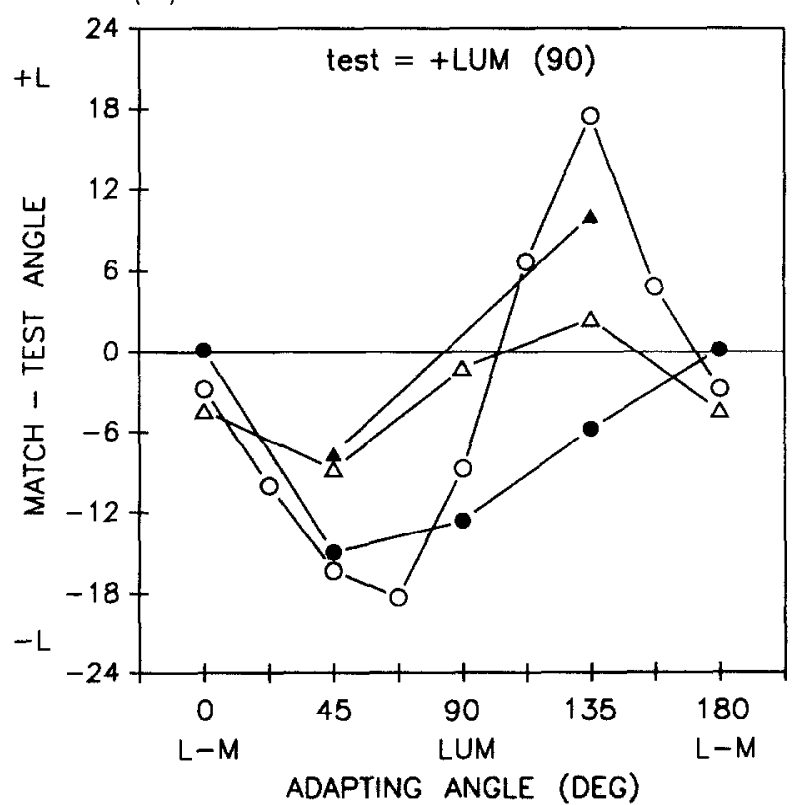

(B)

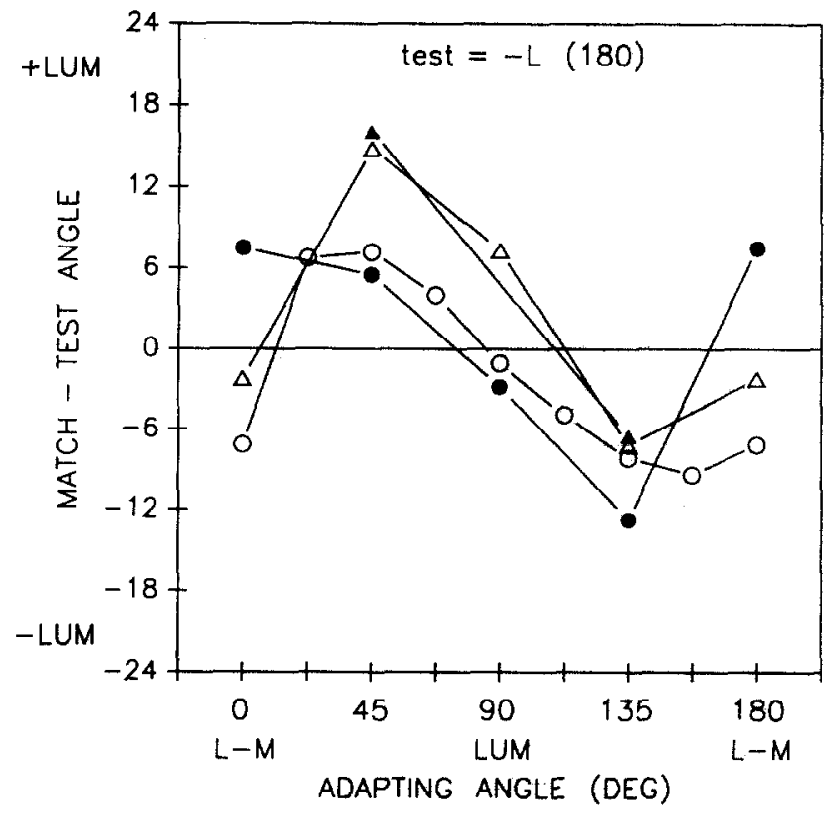

(D)

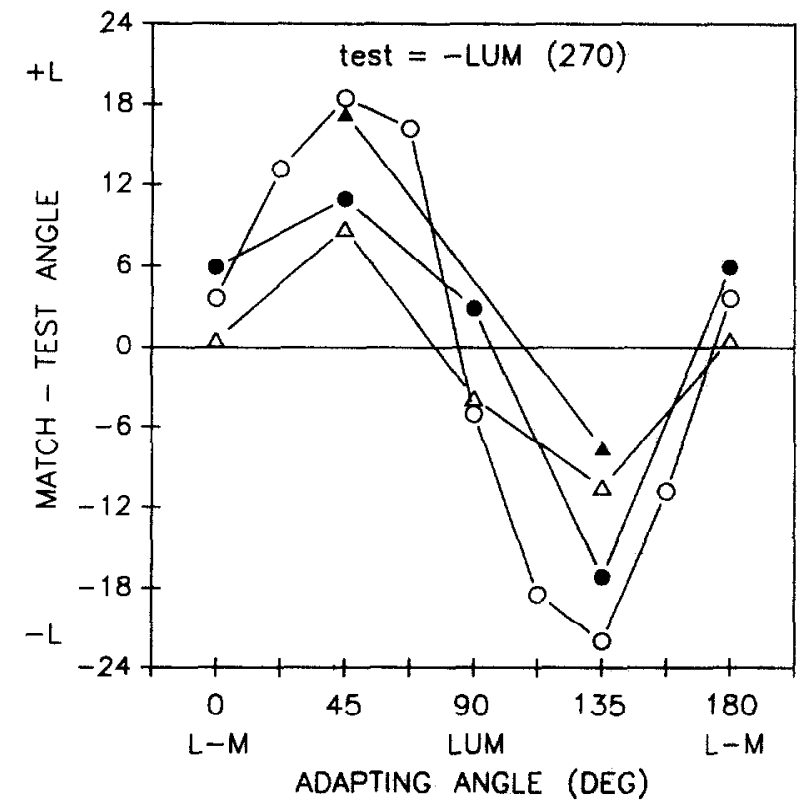

FIGURE 17. Changes in the perceived direction of the four test stimuli that differed from the background only in equiluminant $L-M$ contrast or only in luminance contrast, following adaptation to different directions within the luminance and $L$ - $M$ plane. In each figure the difference between the angles of the match and test coordinates are plotted as a function of the angle of the adapting stimulus for four observers (MW, open circles; JM, solid circles; RV, open triangles; AS, solid triangles, tested only for the 45-225 and $135-315 \mathrm{deg}$ adapting axes). In (A) and (B), positive angle changes indicate that the $L-M$ test was matched by a stimulus that had a higher luminance than the background. In (C) and (D), positive angle changes indicate that the luminance test was matched by a stimulus that had a higher $+\mathrm{L}(-\mathrm{M})$ chromaticity than the background. 


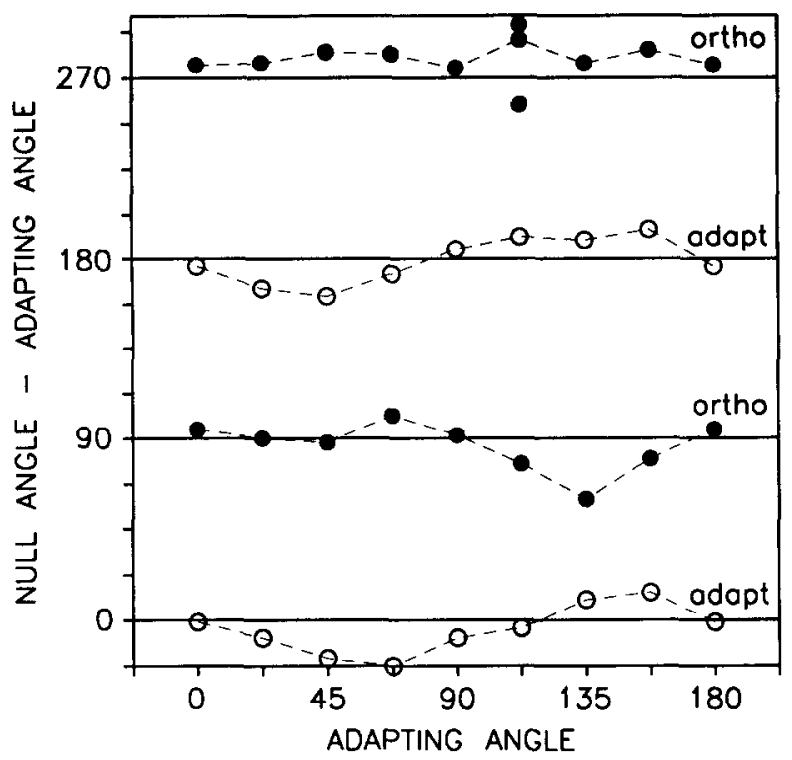

FIGURE 18. Estimates of the axes that did not change in perceived direction following adaptation to different angles within the luminance and L - M plane. Ordinates of " $0 \mathrm{deg}$ " and " $180 \mathrm{deg}$ " correspond to the adapting angle, while values of "90 deg" and " $270 \mathrm{deg}$ " corresponds to angles $\pm 90 \mathrm{deg}$ from the adapting axis. The three nulls near $270 \mathrm{deg}$ for the 112.5-292.5 deg adapting axis are due to the match to the $45 \mathrm{deg}$ test, which was rotated very slightly $(0.3 \mathrm{deg})$ toward the adapting axis (introducing additional zero crossings).

along the orthogonal axes). However, this selectivity was substantially weaker than for either the luminance or the chromatic adapting axis, and thus appeared weaker than the selective interactions suggested by our matching results. [Additional matches to low contrast $(6 \times)$ tests following adaptation to the 45-225 deg axis (contrast $=24 \times$ ) were strongly selective for the adapting axis, suggesting that the weaker selectivity in the reaction times was not due only to the low contrasts examined.]

\section{Interocular transfer of adaptation}

In our final measures we examined whether the color changes produced by different adapting axes exhibit interocular transfer. Measures of threshold changes following adaptation suggest that sensitivity losses transfer for both luminance and chromatic contrast (Krauskopf et al., 1982; Chan, De Valois \& Switkes, 1986), yet colorcontingent aftereffects such as the McCollough effect appear strongly monocular (Stromeyer, 1978). To test for transfer in our task, subjects viewed the display through a haploscope that presented the adapting or the test/matching fields to the same or opposite eyes. The fields were again 2 deg squares centered above (test and adapt) or below (match) fixation. The "unstimulated" eye remained exposed to the background color.

Opposite-eye adaptation to each of the three cardinal directions showed significant transfer as measured by contrast changes along the adapting axis, and the degree of transfer was comparable following luminance or either type of chromatic adaptation. Figure 23 shows matches to equiluminant test stimuli following adaptation to the equiluminant axes of 45-225 or 135-315 deg (in the $S$ vs $\mathrm{L}-\mathrm{M}$ plane rescaled by 1.49 ). For observer $\mathrm{MW}$ the selectivity of the monocular and dichoptic aftereffects appeared qualitatively similar, suggesting that the two conditions differed only in the effective contrast of the adapting stimulus. Surprisingly, however, the second observer showed a significant but largely nonselective color change following dichoptic adaptation, despite clear selectivity for same-eye adaptation. We do not know the bases for these differences; both observers exhibited good stereopsis as assessed informally with random dot stereograms, though we did not attempt more rigorous tests of their binocular vision.

We also tested for binocular interactions in the adapting stimulus by presenting different adapting stimuli to the two eyes. For example, the 45-225 deg adapting stimulus can be decomposed into the component modulations along the $S$ and $L-M$ axes, and these components presented to different eyes. Again, the 135-315 deg adapting stimulus has the same components and thus differed only in the relative phase of the temporal modulation in the two eyes. JP reported that $S$ and $L-M$ components presented to opposite eyes appeared rivalrous, and she yielded matches indistinguishable from the matches for the same-eye adapting component presented alone. Observer MW reported little rivalry (and pronounced fusion) but again exhibited aftereffects that were strongly dominated by the same-eye component, suggesting that there is little binocular integration of the adapting signals.

\section{DISCUSSION}

To summarize, we have found that contrast adaptation to any direction in color-luminance space selectively reduces the perceived contrast of the adapting axis, and biases the perceived hue and lightness of any stimulus away from the adapting axis. Such aftereffects imply that the adapted channels can be selectively tuned to any direction within color-luminance space, with no direction encoded by only a single channel.

The changes in perceived hue we observed are consistent with earlier reports by Guth (1982) and Moxley and Guth (1981), who measured the effects of adapting to an alternation between two monochromatic lights on the perceived hue of monochromatic stimuli. As in the present study, they found that hue changes could be induced in any test stimulus, implying that no chromatic direction invariably isolates only a single post-receptoral chromatic mechanism. Moreover, they similarly argued from the pattern of these hue changes that the sensitivities of the adapted channels differ from the "red-green" and "blue-yellow" dimensions of color appearance.

In many respects our results are also similar to the effects of chromatic contrast adaptation on chromatic detection thresholds that were measured by Krauskopf et al. (1982). In particular, our suprathreshold color changes parallel their threshold results in showing some selectivity for any chromatic direction (Krauskopf $e t$ al., 1986b), but greater selectivity following adaptation to the $\mathbf{L}-\mathbf{M}$ or $\mathbf{S}$ axes. A similar pattern of results has also been reported for measures of the chromatic properties of the 
tilt aftereffect (Flanagan et al., 1990). For some observers in the present study, the selectivity of the color changes for adapting directions intermediate to the $L-M$ and $S$ axes was pronounced. This suggests that mechanisms that can be tuned to these intermediate directions may play a salient role in color appearance.

In the present study we also found pronounced interactions between luminance and chromatic contrast, suggesting that there are channels that can be selectively tuned to specific combinations of luminance and chromatic contrast. In this respect our results differ from the threshold results of Krauskopf et al. (1982), who found instead that adaptation affected luminance and chromatic detection thresholds independently. This difference could reflect either task differences (detection threshold vs suprathreshold appearance), or differences in the stimuli [e.g. $1 \mathrm{~Hz}$ adapt and $0.5 \mathrm{sec}$ square test pulses in the present study compared to $4 \mathrm{~Hz}$ adapt and Gaussian test pulses (50 msec standard deviation) in the study of Krauskopf et al.]. Both of these factors can affect the magnitude of adaptation-dependent interactions be tween luminance and color. Webster and Mollon (1993a) examined how contrast adaptation biased the relative luminosities of two chromaticities [as illustrated in Fig. 17(A, B)], when the luminosities were measured by judging either the perceived lightness, or the flicker or the motion of heterochromatic gratings: adaptation produced large biases in lightness matches and moderate biases in minimum-motion settings for gratings counterphased at $1 \mathrm{~Hz}$, but had little effect on luminosities
(A)

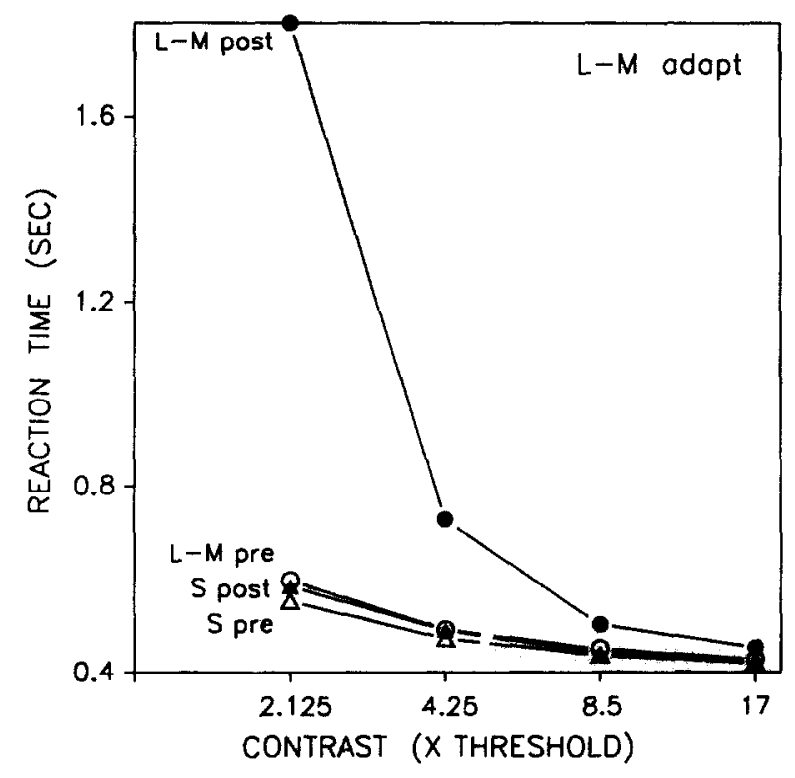

(C)

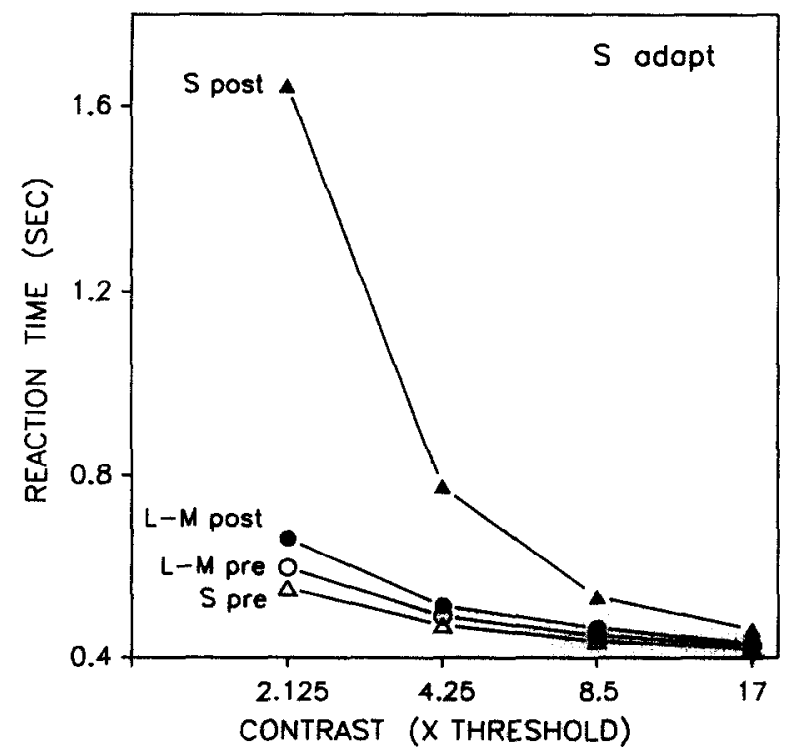

(B)

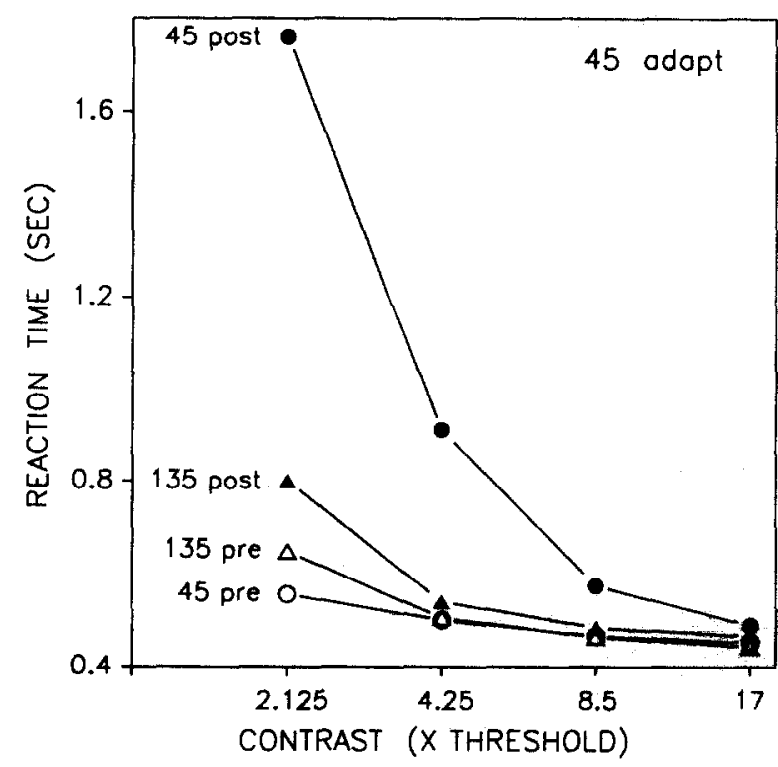

(D)

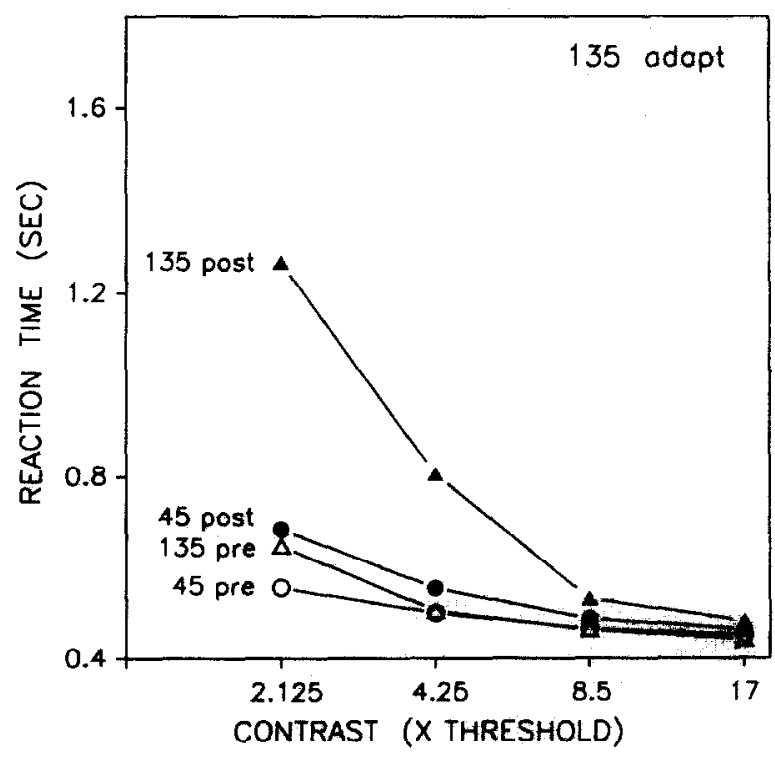

FIGURE 19. Reaction times as a function of test contrast, for stimuli along adapting or orthogonal axes within the $\mathrm{S}$ and $L-M$ plane. The four figures show results for the four different adapting directions tested, either before (pre) or after (post) adaptation. Each point is the average of reaction times for tests on opposite poles of the test axis, and is based on 200 trials. 
(A)

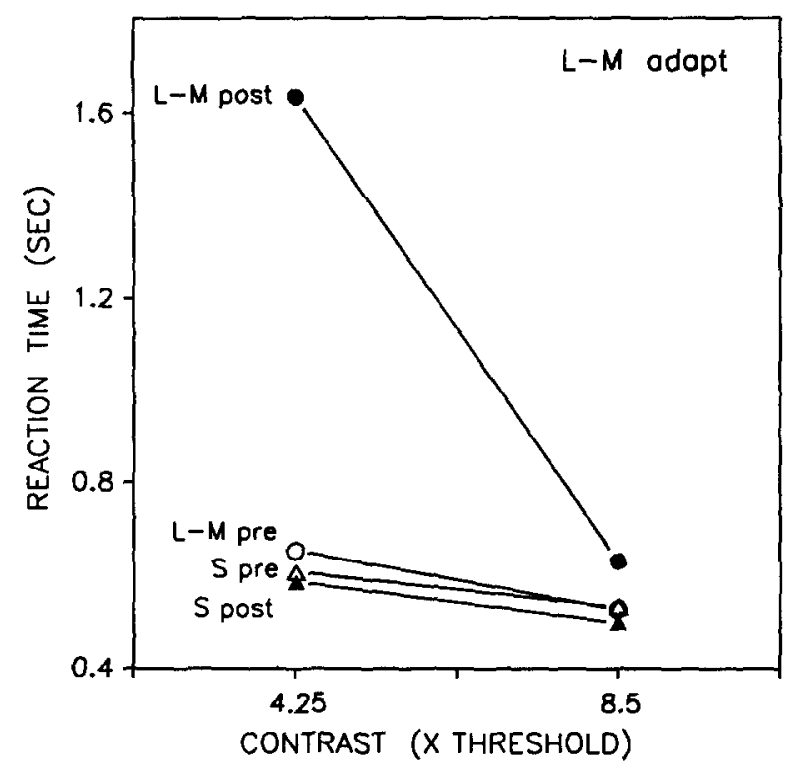

(B)

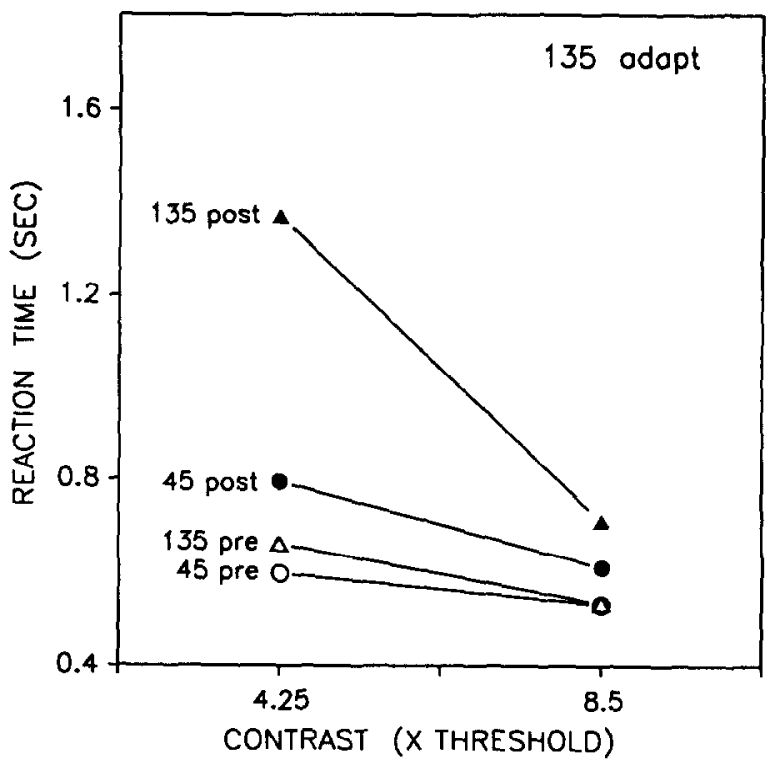

FIGURE 20. Reaction times for equiluminant tests following adaptation to the $\mathbf{L}-\mathbf{M}$ (A) or 135-315 deg (B) adapting axes, for observer JM.

measured by flicker or minimum-motion settings at $15 \mathrm{~Hz}$. These differences are likely to reflect differences in the relative sensitivities to luminance and chromatic contrast under the different measurement conditions (Webster \& Mollon, 1993a). In the present study we also found that interactions between luminance and chromatic contrast appeared substantially weaker when measured by reaction times than when measured by contrast matching. Like minimum-motion or flicker judgments, reaction times for luminance contrast may thus reflect processes that have less sensitivity to chromatic contrast than the processes underlying lightness judgments. For example, such differences could result if lightness judgments depend primarily on the strongly color-opponent parvocellular pathways, while reaction times to luminance contrast depend on magnocellular pathways, whose cells exhibit only weak opponency (Lennie et al., 1993). Gegenfurtner and Kiper (1992) have also observed phasespecific interactions between luminance and chromatic contrast in a masking paradigm, but found that these interactions depended on the spatial properties of the stimulus.

\section{Contrast adaptation and cone-specific adaptation}

Guth (1982) noted that the specific hue changes he measured could also be mimicked by conventional light adaptation, and thus did not unambiguously reflect postreceptoral channels that combine the signals from different cones. For example, von Kries adaptation in cone-selective pathways could alter the appearance of any chromaticity, and these changes would always be relative to the mean adapting chromaticity, even if they depend on independent sensitivity changes within only three cone classes. However, our own contrast matching results cannot be accounted for by cone-specific changes, either in mean sensitivity or in contrast sensitivity. The signature of mean sensitivity changes is an average change in color appearance. Adaptation to modulations of chromaticity around a fixed mean in fact has little effect on average perceived color, and instead affects perceived contrasts relative to the mean. Figure 6 illustrates empirically the qualitatively different effects that light adaptation and contrast adaptation have on color appearance under our stimulus conditions. Shapiro and Zaidi (1992) have also illustrated distinct light adaptation and contrast adaptation effects on detection thresholds.

A contrast adaptation effect within pathways selective for the three classes of cones is inconsistent with the clear selectivity we observed for multiple directions within color-luminance space. Krauskopf et al. (1982) have also noted that cone-specific contrast adaptation is inconsistent with the lack of cross-adaptation between luminance and chromatic contrast. Because the spectral sensitivities of the cones overlap, luminance contrast can produce much larger modulations in the cones than chromatic contrast, yet luminance contrast adaptation has little cffect on chromatic contrast sensitivity (Krauskopf et al., 1982; Bradley et al., 1988).

The task specificity and temporal-frequency specificity of adaptation-induced biases in luminosity matches also argue against a peripheral, cone-specific locus of the sensitivity changes (Webster \& Mollon, 1993a). Moreover, Webster and Mollon (1993a) found that the luminosity biases are spatially selective, while Flanagan $e t$ al. (1989) have reported that perceived hue changes following equiluminant grating adaptation are orientationselective. Similarly, changes in contrast thresholds following either luminance or chromatic contrast adaptation are selective for both spatial frequency and orientation (Gilinsky, 1968; Blakemore \& Campbell, 1969; Bradley et al., 1988). In the present study we have also found that the adaptation-induced changes in color appearance can exhibit strong interocular transfer (though there appeared to be qualitative differences 
among the two observers tested). Krauskopf et al. (1982) and Chan et al. (1986) noted that changes in chromatic threshold sensitivity also exhibit transfer. Such demonstrations of spatial selectivity and interocular transfer are consistent with (but do not prove) a cortical site for the sensitivity changes resulting from contrast adaptation. Supporting this, physiological studies have failed to observe significant contrast adaptation in geniculate cells, while cells in the striate cortex do adapt to contrast (Maffei, Fiorentini \& Bisti, 1973; Ohzawa, Sclar \& Freeman, 1985; Derrington et al., 1984; Sclar, Lennie \& De Priest, 1989).

\section{Multiple channel models of contrast adaptation}

At this presumably central level, we have shown that the conventional model of post-receptoral color vision based on three independent channels fails to account for the changes in color appearance following contrast

(A)

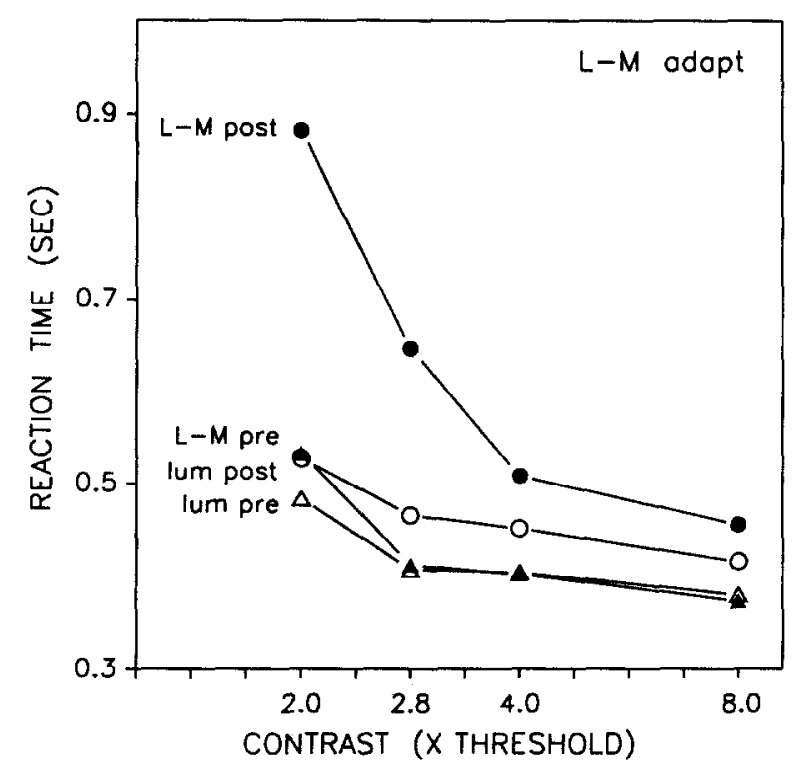

(C)

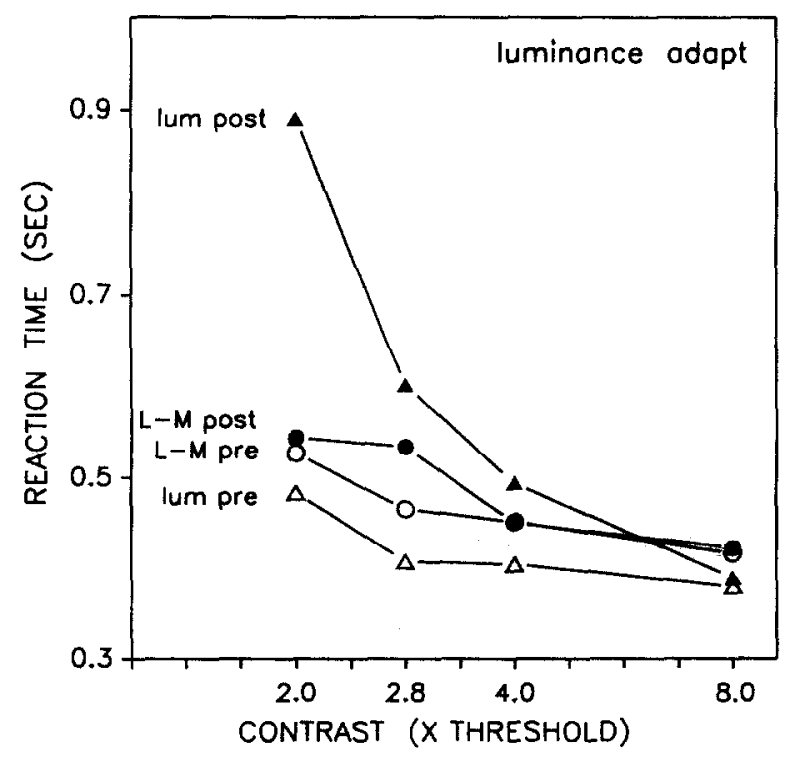

adaptation. However, this model could be modified in at least two different ways to account for our results. One way would be to postulate many more than three independently-adaptable channels, each tuned to at different direction in color space. [We use "independent" adaptation in analogy with cone-specific adaptation, to mean that the sensitivity of one mechanism is uninfluenced by the adaptive state of other mechanisms (Stiles, 1959). This does not imply that the tuning functions of the channels are non-overlapping, or that sensitivity changes in different channels are uncorrelated.] A second way our results could arise is if the channels do not adapt independently, but instead exhibit adaptation-dependent interactions that alter their tuning properties. We consider these two alternatives in turn.

A common assumption of color models is that the selectivity of individual color-luminance mechanisms is

(B)

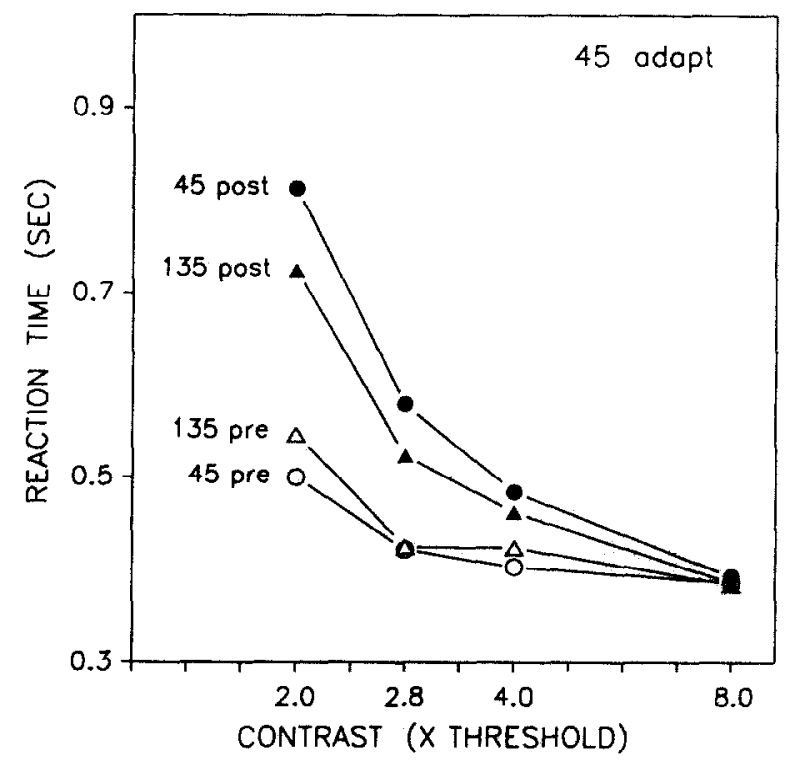

(D)

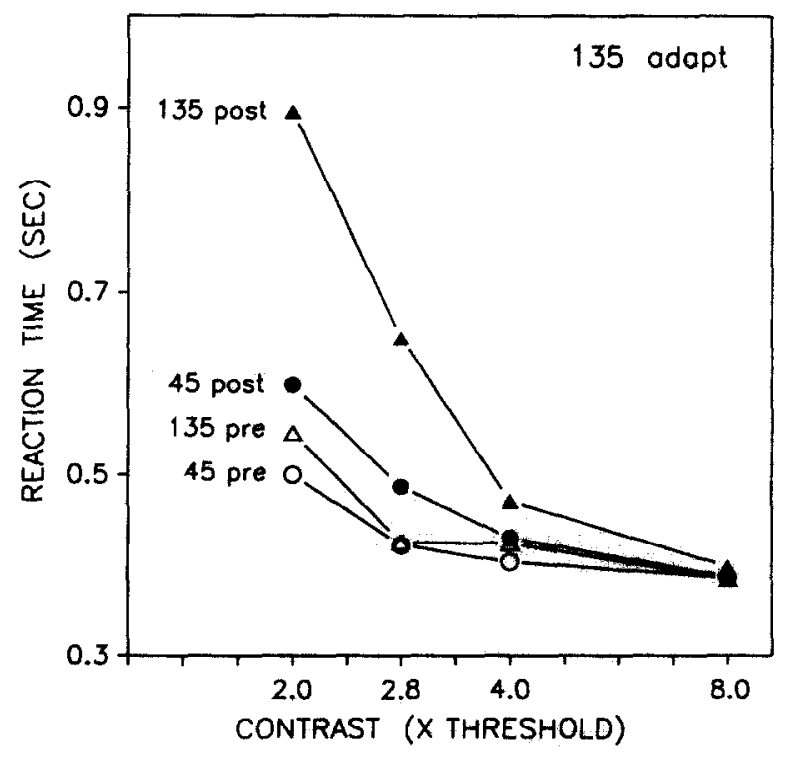

FIGURE 21. Effect of adaptation on reaction times to tests in the luminance and $L-M$ plane. The observer was $M W$. 
(A)

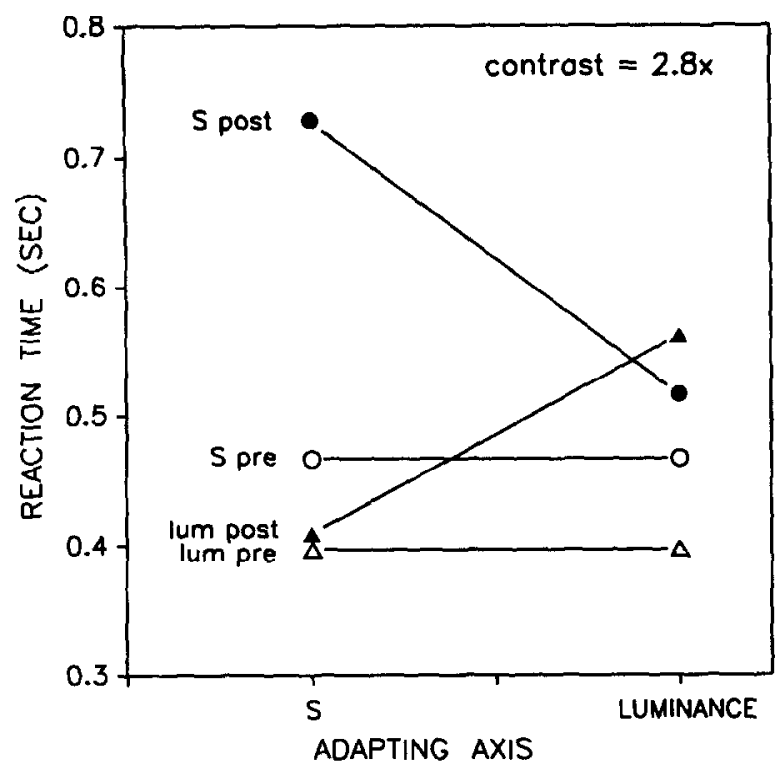

(B)

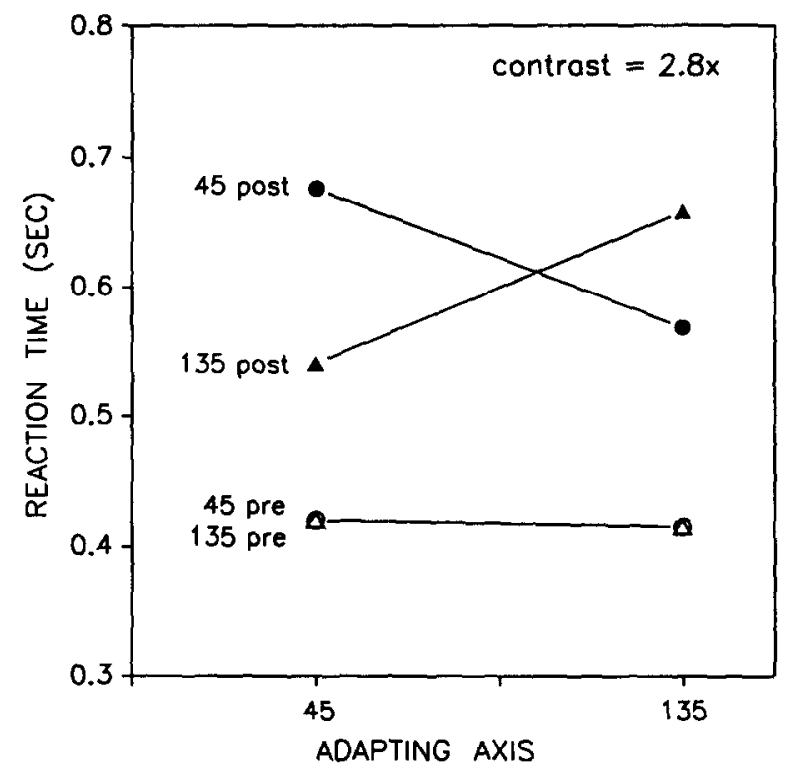

FIGURE 22. Effect of adaptation on reaction times to tests in the luminance and $S$ planes. In these measurements the test contrast was fixed at $2.8 \times$ the pre-adapt threshold. (A) The reaction times before or after either $S$ or luminance adaptation. (B) Corresponding results for the $45-225$ or $135-315 \mathrm{deg}$ adapting and test axes. The observer was MW.

a consequence of different linear combinations of the cone signals. Thus

$$
R_{i}=w_{\mathrm{Li}} \mathrm{L}+w_{\mathrm{Mi}} \mathrm{M}+w_{\mathrm{Si}} \mathrm{S}
$$

where $R_{i}$ is the sensitivity of the $i$ th mechanism, and the $w s$ are the weights attached to the signals from each class of cone. This equation closely characterizes the color selectivities of geniculate cells (Derrington et al., 1984) and of most simple cells in striate cortex (Lennie, Krauskopf \& Sclar, 1990). Within the linear coneexcitation space, it predicts that the relative sensitivity of any mechanism to any axis is given by the cosine of the angle between the axis and the mechanism's preferred direction. The preferred direction thus uniquely specifies the sensitivity of any mechanism (Derrington et al., 1984).

We asked whether the changes in perceived color that we observed could arise from differential adaptation in multiple mechanisms that combine the cone signals linearly. Since the aftereffects appeared most selective for the $\mathbf{L}-\mathbf{M}$ and $\mathbf{S}$ axes, we assumed that the mechanisms' preferred chromatic directions formed two (bipolar) Gaussian distributions centered on the $L-M$ or $S$ directions. [A distribution of this type was suggested by Krauskopf et al. (1982) to account for the residual selectivity for intermediate directions in their threshold task.] Thus the channel density as a function of chromatic angle $(\Theta$, in $\mathrm{deg})$ was given by:

$$
\begin{aligned}
D(\Theta)= & \left\{\exp \left[-1 / 2(\Theta / \sigma)^{2}\right]\right. \\
& \left.+\exp \left[-1 / 2((90-\Theta) / \sigma)^{2}\right]\right\}
\end{aligned}
$$

where $\sigma$ is the standard deviation of the distributions around the $\mathrm{L}-\mathrm{M}(0$ or $180 \mathrm{deg})$ or $\mathrm{S}(90$ or $270 \mathrm{deg})$ axes. (In actual calculations we used a discrete distribution at 1 deg intervals.) We also assumed that color appearance depended on the total responses of the mechanisms along either the $\mathrm{L}-\mathrm{M}$ or the $\mathrm{S}$ dimensions: Perceived direction corresponded to the ratio of $\mathrm{L}-\mathrm{M}$ to $\mathrm{S}$ while perceived contrast corresponded to their vector sum. The total response prior to adaptation was normalized to equal the unadapted test contrast.

To bracket possible forms of the adaptation effect, we modeled the response change due to adaptation either as a multiplicative or a subtractive sensitivity loss. In either case the magnitude of the sensitivity change was assumed to be proportional to the sensitivity of the channel to the adapting axis. Thus the adapted sensitivity, $D_{\text {a }}$, was related to the unadapted sensitivity, $D_{u}$, either by

$$
D_{a}(\Theta)=D_{u}(\Theta)[1-m|\cos (\Theta-\phi)|]
$$

for the multiplicative change, or by

$$
D_{\mathrm{a}}(\Theta)=D_{\mathrm{u}}(\Theta)-m|\cos (\Theta-\phi)|
$$

for the subtractive change, where $\Theta$ and $\phi$ are the preferred channel angle and adapting angle, respectively, and $m$ is the sensitivity change along the adapting axis. The observed adaptation effect measured over a range of test contrasts is intermediate to these two effects (Fig. 11), and thus is not correctly predicted by either model. However, we showed in Figs 7 and 12 that the perceived color changes in the $17 \times$-threshold tests could be closely approximated by multiplicative sensitivity changes. The resulting ellipses rule out a subtractive sensitivity change based on only two mechanisms. However, if the mechanisms form a broad distribution of preferred color directions, then the multiplicative and subtractive hypotheses make similar predictions for the relative color changes along different directions (at a fixed test contrast), since the predicted changes also reflect the pooling of signals across mechanisms. Thus for broad distributions of channels the predicted matches to 
the $17 \times$-threshold tests turn out to be relatively independent of which form of adaptation is assumed.

Finally, stimulus contrasts along the $\mathrm{S}$ axis were rescaled in order to correct for the biases in hue angles and ellipse orientations that we attributed to errors in the relative sensitivities assumed for the $L-M$ and $S$ axes. For three of the observers (MW, AS, and JM) we increased $S$ contrasts by a value of 1.4 , while the matches of the fourth observer (AE) were instead consistent with the original value of 1.0 .

The resulting model had two free parameters: $m$, the sensitivity loss along the adapting axis, and $\sigma$, the standard deviation of preferred directions along the $\mathrm{L}-\mathrm{M}$ or $\mathrm{S}$ axes. If $\sigma$ is very small, then the model reduces to a conventional model with two chromatic channels, one sensitive to the $\mathrm{L}-\mathrm{M}$ signals and the second sensitive to the $\mathrm{S}$ signals (as illustrated in Fig. 1). Alternatively, as $\sigma$ becomes larger the channels approach a uniform distribution, with an equal density at any chromatic direction. We therefore varied the value of $\sigma$ to find the distribution that best accounted for the observed matches. This was done for each observer by varying $\mathrm{m}$ and $\sigma$ to estimate simultaneously the least squares fit of the model to the observed equiluminant matches for all equiluminant adapting axes.

Figures 4 and 5 show that the model provides a good approximation to the color changes for all of the adapting and test directions for each of the observers. In particular, the model closely predicts the sensitivity losses along axes orthogonal to the adapting axis. These sensitivity losses are predicted to occur because the broad spectral tuning predicted by linearly combining

(A)

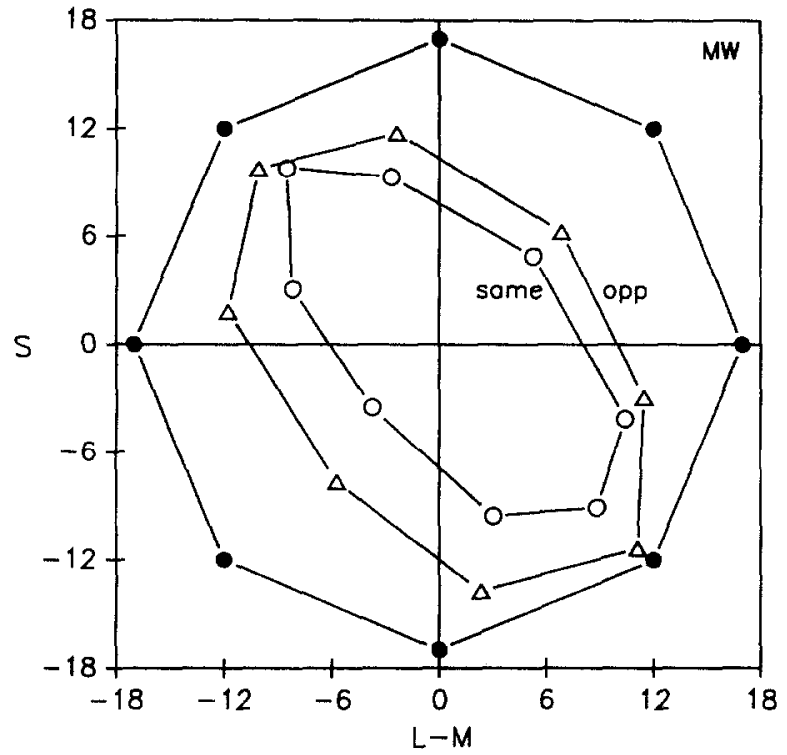

(B)

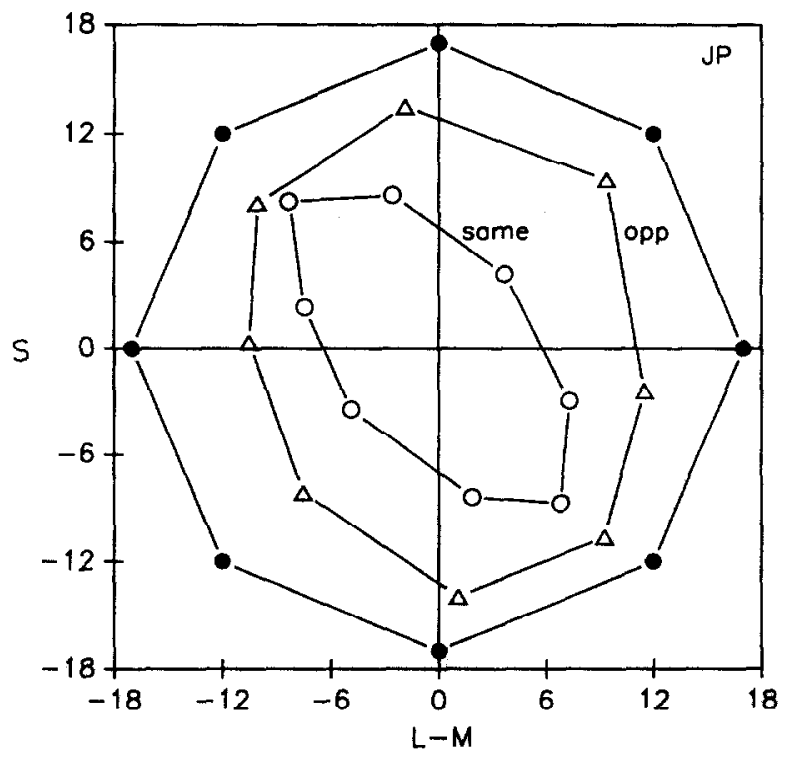

(C)

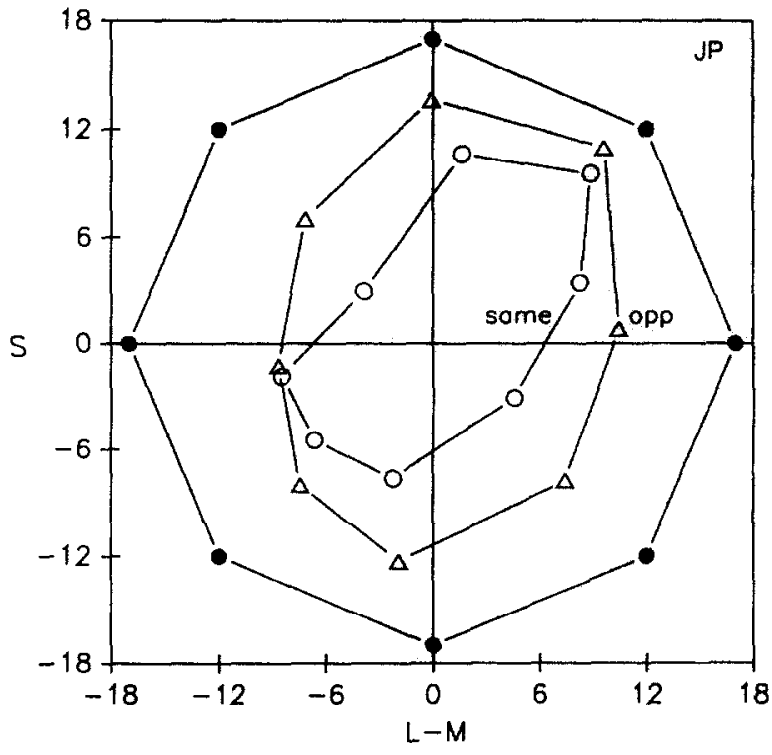

FIGURE 23. Matches in the $S$ and $L-M$ plane for adapting and test stimuli presented to the same or opposite eyes. (A) 45-225 deg adapt, observer MW. (B) 45-225 deg adapt, observer JP. (C) 135-315 deg adapt, observer JP. For these measurements the $S$ contrasts were rescaled to be $1 / 1.49 \times$ the original threshold contrasts. 


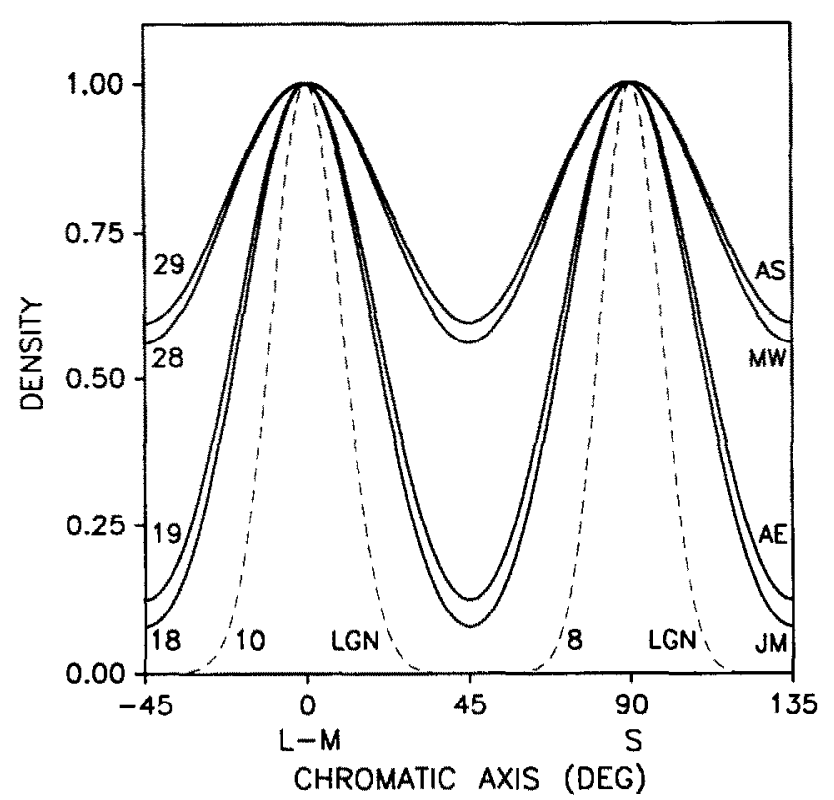

FIGURE 24. Solid lines show the channel distributions estimated from the multiple-channel model fitted to four observers' matches in the $S$ and $L-M$ plane. Standard deviations around the $S$ and $L-M$ axes are indicated on the left of each curve. The best-fitting values of $m$ (corresponding to the magnitude of the adaptation effect) were 0.85 (MW), 0.72 (JM and AE), and 0.66 (AS). Dashed lines show distributions with standard deviations equal to those estimated for parvocellular geniculate neurons by Derrington et al. (1984).

the cones is such that, if more than two channels with different sensitivities span a plane, any adapting and test directions will always excite one or more common channels. For example, a channel with a preferred direction of $45 \mathrm{deg}$ will also have high sensitivity to both the 0 deg and the $90 \mathrm{deg}$ axes $[\cos (45)=0.707]$. Thus this channel should be strongly affected by adaptation to either of these axes, affecting its contribution to the response to the second axis.*

Figure 24 plots the best-fitting distribution of channels for each of the four observers tested. In these distributions the standard deviation of preferred directions around the $\mathrm{L}-\mathrm{M}$ or $\mathrm{S}$ axes ranges from 18 to $29 \mathrm{deg}$. Thus substantial variability was required to account for the matches of all observers. On the other hand, sensitivity biases along the $L-M$ and $S$ axes are clear, though for MW and AS these resulted in only modest improvements in the fits over a uniform distribution. Figure 24 also plots the channel distribution based on two Gaussians that have the same standard deviations as cells in the parvocellular geniculate. These have values of $10 \mathrm{deg}(\mathrm{L}-\mathrm{M})$ and $8 \mathrm{deg}(\mathrm{S})$ based on the estimates of Derrington et al. (1984, see their Fig. 15), converted into

\footnotetext{
${ }^{*}$ Krauskopf et al. (1982) observed little threshold elevation when adapting and test stimuli were contined to different cardinal axes, though weak cross-adaptation effects have been found at threshold for luminance and chromatic gratings (Bradley et al.. 1988). Detection thresholds might exhibit weaker cross adaptation effects than our suprathreshold contrast changes if the thresholds depend more strongly on the responses of the most sensitive mechanisms to the test, and thus less strongly on the pooling of signals across mechanisms that our suprathreshold model assumes.
}

the units of our color space. The geniculate distribution is narrower than the distribution calculated for any of our observers, and this difference is pronounced for two of the observers (AS and MW). Our results could thus refiect the wider range of chromatic preferences reported for striate cortical cells (Lennie et al., 1990; Elfar \& De Valois, 1993), and in this sense are consistent with the "higher-order" transformation of color mechanisms suggested by Krauskopf et al. (1986b). (Note that this analysis indicates nothing about the relative heights of the distributions along the $\mathrm{S}$ and $\mathrm{L}-\mathrm{M}$ axes, since sensitivities along these two directions have been equated.)

\section{Channel interaction models of contrast adaptation}

In the preceding model we assumed that adaptation occurs independently within each channel, and does not alter the selectivity of individual channels. However, Barlow (1989) and Barlow and Földiák (1989) suggested that contrast adaptation might also induce interactions between channels, and that these interactions change the stimulus dimensions to which the channels are tuned. They showed that such interactions could lead to more efficient coding in the visual system, by making the responses of the channels to the prevailing ensemble of stimuli statistically independent. The interaction they postulated was reciprocal inhibitory feedback between two channels that builds up whenever the channels' responses to the stimuli are correlated. For example, for two channels, the interactions proposed by Barlow and Földiák are of the form

$$
\begin{aligned}
& O_{1}=I_{1}-w O_{2} \\
& O_{2}=I_{2}-w O_{1}
\end{aligned}
$$

where $I_{1}$ and $I_{2}$ are the inputs to the channels, and $O_{1}$ and $\mathrm{O}_{2}$ are the outputs. (The outputs are assumed to be normalized to unit variance by a multiplicative gaincontrol.) The response of each channel thus depends on its own input minus a proportion $(w)$ of the second channel's response, and adaptation is assumed to adjust the value of $w$ until $O_{1}$ and $O_{2}$ are decorrelated.

We noted previously that interactions of the type proposed by Barlow and Földiák could provide an alternative basis for the changes in color appearance we observed following adaptation (Webster \& Mollon, 1991). For example, inhibition between two chromatic channels would result in an oblique rotation of the channels' response axes. This could selectively reduce sensitivity to adapting axes to which neither individual channel is tuned, and stimuli that initially isolated one of the channels would now induce a response of opposite sign in the second channel, changing the distribution of responses to such stimuli.

Recently Atick, Li and Redlich (1993) reported a model of adaptive decorrelation which they showed can closely describe the color-luminance matches we initially reported (Webster \& Mollon, 1991). Their algorithm decorrelates and normalizes the responses of two 
channels to the adapting stimulus through the following rotation and scaling transformations:

$$
\begin{array}{r}
\left|\begin{array}{c}
O_{\mathrm{a} 1} \\
O_{\mathrm{a} 2}
\end{array}\right|=\left|\begin{array}{rr}
\cos \Theta & -\sin \Theta \\
\sin \Theta & \cos \Theta
\end{array}\right| \cdot\left|\begin{array}{cc}
m_{1} & 0 \\
0 & m_{2}
\end{array}\right| . \\
\times\left|\begin{array}{cc}
\cos \Theta & \sin \Theta \\
-\sin \Theta & \cos \Theta
\end{array}\right| \cdot\left|\begin{array}{l}
O_{\mathrm{u} 1} \\
O_{\mathrm{u} 2}
\end{array}\right|
\end{array}
$$

where $\Theta$ is the adapting angle, and $m_{1}$ and $m_{2}$ are the multiplicative gain changes along the adapting and orthogonal axes, respectively. This perceptually distorts the initial circle of test coordinates into an ellipse whose minor axis is oriented along the adapting axis, and whose axis lengths (for appropriate choices of $m_{1}$ and $m_{2}$ ) equal the perceived contrasts along the adapting and orthogonal axes. Figures 7 and 12 show that such ellipses closely characterize both the perceived contrast changes and perceived hue changes in our $17 \times$-threshold tests. To account for the contrast changes along the orthogonal axis, Atick et al. suggested that the visual response to a single adapting axis may be noisy, and this noise could mimic the presence of stimulus modulations along the orthogonal axis. However, this implies a low signal to noise ratio in the channel responses (Atick et al., 1993), and the sensitivity losses along orthogonal axes following adaptation to covarying luminance and chromatic contrasts are not substantially weaker than the losses following adaptation to uncorrelated variations in luminance and chromaticity [Fig. 14(C)].

While these models of decorrelation assume a multiplicative adaptation effect, response changes of this form are not clearly evident psychophysically. Instead, our results are consistent with other studies of contrast adaptation in suggesting that adaptation has proportionately weaker effects on stimuli of higher contrast, and typically has only weak or no effect on the appearance of contrasts that equal or exceed the adapting contrast (Blakemore et al., 1971; Georgeson, 1985; Webster, De Valois \& Switkes, 1987; Shapiro \& Zaidi, 1992; present study, Fig. 11). This proportional loss of an adaptation effect at higher contrasts may thus constrain the extent to which the channels arc decorrelated following adaptation. Recently Snowden and Hammett (1992) reported evidence for distinct subtractive and multiplicative contrast adaptation effects, which they attributed to response changes within channels (subtractive, and revealed when adapting and test gratings have the same orientation) and interactions between channels (multiplicative, and revealed when adapting and test orientations differ). As we noted, the contrast changes we measured along the adapting and orthogonal orientations in color space do not follow either of these functional forms. Yet the differcnces between the sensitivity changes along the adapting and orthogonal axes are not predicted by either of the two models we have considered.

The model of Atick et al. demonstrates how interactions between only three post-receptoral mechanisms can potentially account for all of the selective changes in color appearance we measured, and our results do not allow us to discriminate between this class of model and the alternative of multiple independently-adaptable channels (or interactions between multiple channels). However, it remains questionable whether three channels can ultimately characterize post-receptoral color vision. First, the assumption of three discrete channels ignores the demonstrated variability in the spectral tuning of individual neurons in the lateral geniculate (e.g. De Valois, Jacobs \& Abramov, 1966; Derrington et al., 1984), and particularly in striate cells (Lennie et al., 1990; Elfar \& De Valois, 1993), which exhibit contrast adaptation. Second, Krauskopf et al. (1986b) demonstrated selectivity for more than two chromatic directions even in a neutral state of adaptation, by showing that two colors defined by axes intermediate to the cardinal axes (e.g. a pair of colors at 45 and $135 \mathrm{deg}$ ) were discriminable at the detection threshold. Conversely, there appear to be few studies that provide unequivocal evidence for only three discrete mechanisms. Channels tuned to only three linearly independent dimensions are sufficient to account for luminance and chromatic discrimination thresholds under a wide variety of conditions (Lennie et al., 1993). Yet the trichromacy of the cones necessarily limits sensitivity to only three dimensions, and many of these results could also be plausibly accounted for by assuming that these three dimensions are represented by many post-receptoral mechanisms with different spectral sensitivities. The differences in selectivity for diffcrent adapting directions suggest that some stimulus dimensions have a special status in the post-receptoral organization of the cone signals, and that the distribution of channel sensitivities is not uniform, yet this does not imply the truly discrete representation that is conventionally assumed.

The profound changes that contrast adaptation induces in color appearance suggest that contrast adaptation effects could influence the capacity for color constancy (i.e. the ability to form stable representations of surface reflectance functions under different viewing conditions). The role of contrast adaptation in constancy judgments remains unexplored, for studies of color constancy have typically focused on how color appearance is affected by mean changes in illuminants, and how these mean changes can be compensated for by processes of light adaptation (Pokorny, Shevell \& Smith, 1992). However, different illuminants can also alter the set of contrasts present within the image, and alter the correlations between different dimensions in color-luminance space, and such changes cannot in general be factored out by mean changes in visual sensitivity. Eye movements over different regions of a scene, which might serve to light adapt the observer to the average luminance and chromaticity in the image (e.g. Fairchild \& Lennie, 1992), are also likely to generate the temporal modulations in luminance and chromaticity that characterize our adapting stimuli. Moreover, studies of simultaneous induction effects have shown that perceived color and lightness depend strongly on the range of spatial contrasts in the image (Chubb, Sperling \& 
Solomon, 1989; Brown \& Macleod, 1991; Singer, Han \& D'Zmura, 1993). While both light adaptation and contrast adaptation can significantly influence color perception, little is known about how these distinct processes might interact to regulate visual sensitivity, a question we are currently pursuing (Webster \& Mollon, 1993b).

\section{REFERENCES}

Atick, J. J., Ii, Z. \& Redlich, A. N. (1993). What does post-adaptation color appearance reveal about cortical color representation. Vision Research, 33, 123-129.

Barlow, H. B. (1989) A theory about the functional role and synaptic mechanism of visual after-effects. In Blakemore, C. (Ed.), Vision: Coding and efficiency. Cambridge: Cambridge University Press.

Barlow, H. B. \& Földiák, P. F. (1989). Adaptation and decorrelation in the cortex. In Durbin, R., Miall, C. \& Mitchison, G. J. (Eds), The computing neuron. Reading, Mass.: Addison-Wesley.

von Békésy, G. (1929). Zur Theorie des Hörens; Über die Bestimmung des einem reinen Tonempfinden entsprechenden Erregungsgebietes der Basilarmembran vermittelst Ermüdungserscheinungen. Physikalische Zeitschrift, 30, 115-125. [Translated into English by Wever, E. G. in von Békésy, G. (1960). Experiments in hearing (Trans.) (pp. 354 -368). New York: McGraw-Hill.]

Benzschawel, T. \& Guth, S. L. (1982). Post-receptor chromatic mechanisms revealed by flickering vs fused adaptation. Vision Research, $22,69-75$.

Blakemore, C. \& Campbell, F. W. (1969). On the existence of neurones in the human visual system selectively sensitive to the orientation and size of retinal images. Journal of Physiology, London, 203, $237-260$.

Blakemore, C. \& Sutton, P. (1969). Size adaptation: A new aftereffect. Science, 166, 245-247.

Blakemore, C., Muncey, J. P. J. \& Ridley, R. M. (1971). Perceptual fading of a stabilized cortical image. Nature, 233, 204-205.

Boynton, R. M. \& Kambe, N. (1980). Chromatic difference steps of moderate size measured along theoretically critical axes. Color Research and Application, 5, 13-23.

Bradley, A., Switkes, E. \& De Valois, K. K. (1988). Orientation and spatial frequency selectivity of adaptation to color and luminance gratings. Vision Research, 28, 841-856.

Brown, R. O. \& MacLeod, D. I. A. (1991). Induction and constancy for color saturation and achromatic contrast variance. Investigative Ophthalmology and Visual Science (Suppl.), 32, 1214.

Burns, S. A., Elsner, A. E., Pokorny, J. \& Smith, V. C. (1984). The Abney effect: Chromaticity of unique and other constant hues. Vision Research, 24, 479-489.

Chan, H., De Valois, K. K. \& Switkes, E. (1986). Binocular spatial interactions of equiluminant color and luminance patterns. Investigative Ophthalmology and Visual Science (Suppl.), 27, 75.

Cole, G. R., Hine, T. \& McIlhagga, W. (1993). Detection mechanisms in L-, M-, and S-cone contrast space. Journal of the Optical Society of America $A, 10,38-51$.

Chubb, C., Sperling, G. \& Solomon, J. A. (1989). Texture interactions determine perceived contrast. Proceedings of the National Academy of Science, U.S.A., 86, 9631-9635.

Derrington, A. M., Krauskopf, J. \& Lennie, P. (1984). Chromatic mechanisms in lateral geniculate nucleus of macaque. Journal of Physiology, 357, 241-265.

De Valois, R. L., Abramov, I. \& Jacobs, G. H. (1966). Analysis of response patterns of LGN cells. Journal of the Optical Society of America, 56, 966-977.

De Valois, R. L., Snodderly, D. M. Jr, Yund, E. W. \& Hepler, N. K. (1977). Responses of macaque lateral geniculate cells to luminance and color figures. Sensory Processes, 1, 244-259.

D'Zmura, M. (1991). Color in visual search. Vision Research, 31, 951-966.

Elfar, S. D. \& De Valois, R. L. (1993). Chromatic tuning propertics of monkey striate cells. Investigative Ophthalmology and Visual Science (Suppl.), 34, 793.
Fairchild, M. D. \& Lennie, P. (1992). Chromatic adaptation to natural and incandescent illuminants. Vision Research, 32, 2077-2085.

Flanagan, P., Cavanagh, P. \& Crassini, B. (1989). McCollough effects for equiluminant gratings. Investigative Ophthalmology and Visual Science (Suppl.), 30, 130.

Flanagan, P., Cavanagh, P. \& Favreau, O. E. (1990). Independent orientation-selective mechanisms for the cardinal directions of colour space. Vision Research, 30, 769-778.

Gegenfurtner, K. \& Kiper, D. C. (1992). Contrast detection in luminance and chromatic noise. Journal of the Optical Society of America $A, 9,1880-1888$.

Georgeson, M. A. (1985). The effect of spatial adaptation on perceived contrast. Spatial Vision, I, 103-112.

Gibson, J. J. \& Radner, M. (1937). Adaptation, after-effect and contrast in the perception of tilted lines. I. Quantitative studies. Journal of Experimental Psychology, 20, 453-467.

Gilinsky, A. S. (1968). Orientation-specific effects of patterns of adapting light on visual acuity. Journal of the Optical Society of America, 58, 13-17.

Guth, S. L. (1982). Hue shifts following flicker vs. fused adaptation reveal initial opponent mechanisms. Investigative Ophthalmology and Visual Science (Suppl.), 22, 78.

Guth, S. L. \& Moxley, J. P. (1982). Hue shifts following differential postreceptor achromatic adaptation. Journal of the Optical Society of America, 72, 301-303.

Henmon, V. A. C. (1906). The time of perception as a measure of differences in sensations. Archives of Philosophy, Psychology, and Scientific Method, No. 8, 1-75.

Hurvich, L. M. \& Jameson, D. (1957). An opponent-process theory of color vision. Psychological Review, 64, 384-404.

Jameson, D. \& Hurvich, L. M. (1972). Color adaptation: Sensitivity, contrast, after-images. In Jameson, D. \& Hurvich, L. M. (Eds), Handbook of sensory physiology (Vol. 7, Part 4). New York: Springer.

Krauskopf, J. \& Farell, B. (1990). Influence of colour on the perception of coherent motion. Nature, 348, 328-331.

Krauskopf, J. \& Gegenfurtner, K. (1992). Color discrimination and adaptation. Vision Research, 32, 2165-2175.

Krauskopf, J., Williams, D. R. \& Heeley, D. W. (1982). Cardinal directions of color space. Vision Research, 22, 11231131.

Krauskopf, J., Zaidi, Q. \& Mandler, M. B. (1986a). Mechanisms of simultaneous color induction. Journal of the Optical Society of America A, 3, 1752-1757.

Krauskopf, J., Williams, D. R., Mandler, M. B. \& Brown, A. M. (1986b). Higher order color mechanisms. Vision Research, 26, $23-32$.

Le Grand, Y. (1949). Les seuils différentials de couleurs dans la théorie de Young. Revue d'optique théorique et instrumentale, 28, 261-278.

Lennie, P. \& D'Zmura, M. (1988). Mechanisms of color vision. CRC Critical Reviews in Neurobiology, 3, 333-400.

Lennie, P., Krauskopf, J. \& Sclar, G. (1990). Chromatic mechanisms in striate cortex of macaque. Journal of Neuroscience, 10, 649-669.

Lennie, P., Pokorny, J. \& Smith, V. C. (1993). Luminance. Journal of the Optical Society of America A, 10, 1283-1293.

MacLeod, D. I. A. \& Boynton, R. M. (1979). Chromaticity diagram showing cone excitation by stimuli of equal luminance. Journal of the Optical Society of America, 69, 1183-1186.

Maffei, L., Fiorentini, A. \& Bisti, S. (1973). Neural correlate of perceptual adaptation to gratings. Science, 182, 1036-1038.

Mollon, J. D. (1989). "Tho' she knecl'd in that Place where they grew. .". Journal of Experimental Biology, 146, 21-38.

Moxley, J. P. \& Guth, S. L. (1981). Hue shifts caused by post-receptor adaptation. Investigative Ophthalmology and Visual Science (Suppl.), $20,206$.

Moxley, J. P., Guth, S. L. \& Geisler, W. S. (1979). Hue shifts reveal postreceptor effects: Data and demonstration. Investigative Ophthalmology and Visual Science (Suppl.), 208.

Nagy, A. L. \& Sanchez, R. R. (1990). Critical color differences determined with a visual search task. Journal of the Optical Society of America A, 7, 1209-1217. 
Ohzawa, I., Sclar, G. \& Freeman, R. D. (1985). Contrast gain control in the cat's visual system. Journal of Neurophysiology, 54 , $651-667$.

Osgood, C. E. \& Heyer, A. W. (1952). A new interpretation of figural after-effects. Psychological Review, 59, 98-118.

Pantle, A. (1972). Flicker adaptation-Il. Effect on the apparent brightness of intermittent lights. Vision Research, 12, 705-715.

Pokorny, J., Shevell, S. K. \& Smith, V. C. (1992). Colour appearance and colour constancy. In Gouras, P. (Ed.), Vision and visual dysfunction 6: The perception of colour. London: Macmillan.

Sclar, G., Lennie, P. \& DePriest, D. D. (1989). Contrast adaptation in striate cortex of macaque. Vision Research, 29, 747-755.

Shapiro, A. G. \& Zaidi, Q. (1992). The effects of prolonged temporal modulation on the differential response of color mechanisms. Vision Research, 32, 2065-2075.

Singer, B., Han, K.-H. \& D'Zmura, M. (1993). Color selectivity in contrast gain control. Investigative Ophthalmology and Visual Science, 34, 764.

Snowden, R. J. \& Hammett, S. T. (1992). Subtractive and divisive adaptation in the human visual system. Nature, 355, 248-250.

Stiles, W. S. (1959). Color vision: The approach through incrementthreshold sensitivity. Proceedings of the National Academy of Science, 45, 100-114.

Stromeyer, C. F. III (1978). Form-color aftereffects in human vision. In Held, R., Leibowitz, H. W. \& Teuber, H. L. (Eds), Handbook of sensory physiology (Vol. 8). New York: Springer.

Stromeyer, C. F. III \& Lee, J. (1988). Adaptational effects of short wave cone signals on red-green chromatic detection. Vision Research, $28,931-940$.
Webster, M. A. \& Mollon. J. D. (1991). Changes in colour appearance following post-receptoral adaptation. Nature, 349. 235238.

Webster, M. A. \& Mollon, J. D. (1993a). Contrast adaptation dissociates different measures of luminous efficiency. Iom of thal Optical Society of America A, 10, 13321340

Webster, M. A. \& Mollon, J. D. (1993b). Light adaptation and contrast adaptation. Perception (Supp/), 22, 13 14

Webster, M. A., De Valois, K. K. \& Switkes, E. (1987). Effect of contrast adaptation on color and luminance interactions. Investigative Ophthalmology and Visual Science (Suppl.). 28, 214.

Webster, M. A., De Valois, K. K. \& Switkes, E. (1990). Orientation and spatial-frequency discrimination for luminance and chromatic gratings. Journal of the Optical Society of America A, 7, 1034-1049.

Wiesel. T. N. \& Hubel, D. H. (1966). Spatial and chromatic interactions in the lateral geniculate body of the rhesus monkey. Journal of Neurophysiology, 29, 1115-1156.

Wohlgemuth, A. (1911). On the after-effect of seen movement. British Journal of Psychology Monograph Supplements, No. 1, 1 117.

Zaidi, Q. \& Halevy, D. (1993). Visual mechanisms that signal the direction of color changes. Vision Research, 33. 1037.1051.

Acknowledgements - We thank Anya Ekstein, Jeanette Payne, Alex Shepherd, and Renee Van Der Most for participating as observers. Supported by the SERC and the Isaac Newton Trust. Parts of this work were carried out while MAW was supported by fellowships from NIH and NSF (NATO), and by a Private Patients Plan Visiting Fellowship in Medicine at Jesus College. University of Cambridge. 